\title{
Source Code Analysis Laboratory (SCALe)
}

Robert C. Seacord

William Dormann

James McCurley

Philip Miller

Robert Stoddard

David Svoboda

Jefferson Welch

April 2012

TECHNICAL NOTE

CMU/SEI-2012-TN-013

\section{CERT $^{\circledR}$ Program}

Unlimited distribution subject to the copyright.

http://www.cert.org

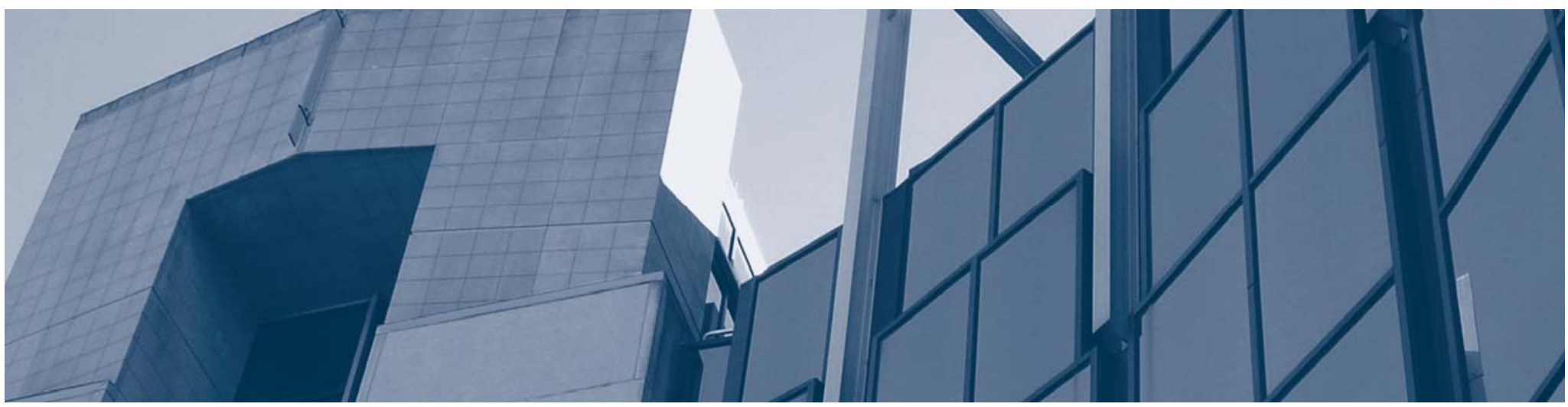


Copyright 2012 Carnegie Mellon University.

This material is based upon work funded and supported by the United States Department of Defense under Contract No. FA8721-05-C-0003 with Carnegie Mellon University for the operation of the Software Engineering Institute, a federally funded research and development center.

Any opinions, findings and conclusions or recommendations expressed in this material are those of the author(s) and do not necessarily reflect the views of the United States Department of Defense.

This report was prepared for the

Contracting Officer

ESC/CAA

20 Shilling Circle

Building 1305, 3rd Floor

Hanscom AFB, MA 01731-2125

NO WARRANTY

THIS CARNEGIE MELLON UNIVERSITY AND SOFTWARE ENGINEERING INSTITUTE MATERIAL IS FURNISHED ON AN “AS-IS” BASIS. CARNEGIE MELLON UNIVERSITY MAKES NO WARRANTIES OF ANY KIND, EITHER EXPRESSED OR IMPLIED, AS TO ANY MATTER INCLUDING, BUT NOT LIMITED TO, WARRANTY OF FITNESS FOR PURPOSE OR MERCHANTABILITY, EXCLUSIVITY, OR RESULTS OBTAINED FROM USE OF THE MATERIAL. CARNEGIE MELLON UNIVERSITY DOES NOT MAKE ANY WARRANTY OF ANY KIND WITH RESPECT TO FREEDOM FROM PATENT, TRADEMARK, OR COPYRIGHT INFRINGEMENT.

This material has been approved for public release and unlimited distribution except as restricted below.

Internal use:* Permission to reproduce this material and to prepare derivative works from this material for internal use is granted, provided the copyright and "No Warranty" statements are included with all reproductions and derivative works.

External use:* This material may be reproduced in its entirety, without modification, and freely distributed in written or electronic form without requesting formal permission. Permission is required for any other external and/or commercial use. Requests for permission should be directed to the Software Engineering Institute at permission@sei.cmu.edu.

( $\quad$ Carnegie Mellon and CERT are registered in the U.S. Patent and Trademark Office by Carnegie Mellon University.

TM Carnegie Mellon Software Engineering Institute (stylized), Carnegie Mellon Software Engineering Institute (and design), Simplex, and the stylized hexagon are trademarks of Carnegie Mellon University.

* These restrictions do not apply to U.S. government entities. 


\section{Table of Contents}

Acknowledgments vii

$\begin{array}{ll}\text { Abstract ix } & \text { ix }\end{array}$

1 Introduction 1

1.1 Software Security 1

1.2 SCALe 1

1.3 Conformance Assessment 2

1.4 CERT Secure Coding Standards 3

1.5 Automated Analysis Tools 4

1.5.1 Static Analysis Tools 4

1.5.2 Dynamic Analysis and Fuzz Testing 5

1.6 Portability and Security 5

$\begin{array}{ll}1.7 & \text { SCALe for Varying Application Domains }\end{array}$

2 SCALe Process $\quad 8$

2.1 Conformance Testing Outcomes 8

2.2 SCALe Laboratory Environment 9

$\begin{array}{lll}2.3 & \text { Conformance Testing Process } & 10\end{array}$

2.4 The Use of Analyzers in Conformance Testing 11

2.5 Conformance Test Results 13

2.5.1 Conformance Test Results Generation 13

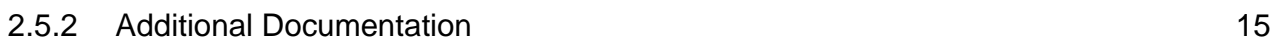

2.6 Tracking Diagnostics Across Code Base Version 16

2.6.1 Standard Approach 18

$\begin{array}{ll}2.7 & \text { Quality Control } \\ & 19\end{array}$

$\begin{array}{lll}2.7 .1 & \text { Personnel } & 19\end{array}$

$\begin{array}{ll}\text { 2.7.2 Quality Assurance Procedures } & 20\end{array}$

$3 \quad$ Conformance Testing 28

3.1 Introduction 28

$\begin{array}{lll}3.1 .1 & \text { Impartiality } & 29\end{array}$

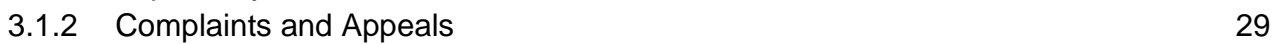

$\begin{array}{lr}3.1 .3 & 29\end{array}$

\begin{tabular}{ll}
3.2 & CERT SCALe Seal \\
\hline 3.3 & 29
\end{tabular}

3.3 CERT SCALe Service Agreement 30

3.3.1 Conformance Certificates 30

3.4 SCALe Accreditation 31

3.5 Transition 31

3.6 Conformance Test Results 32

$\begin{array}{lll}\text { 3.6.1 System A } & 32\end{array}$

$\begin{array}{lll}3.6 .2 & \text { System B } & 34\end{array}$

$4 \quad$ Related Efforts $\quad 36$

4.1 Veracode 36

4.2 ICSA Labs 36

4.3 SAIC Accreditation and Certification Services 36

4.4 The Open Group Product Certification Services 36 
$5 \quad$ Future Work and Summary

5.1 Future Work

5.2 Summary

38

Bibliography 


\section{List of Figures}

Figure 1: $\quad$ SCALe Process Overview 2

Figure 2: $\quad$ C and C++ "Breadth" Case Coverage [Landwehr 2008] 5

Figure 3: $\quad$ Source Code Analysis Laboratory $\quad 9$

Figure 4: $\quad$ SCALe Conformance Testing Process 10

Figure 5: $\quad$ Attribute Agreement Analysis Using Minitab 26

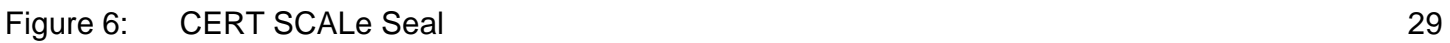


CMU/SEI-2012-TN-013 | iv 


\section{List of Tables}

Table 1: $\quad$ True Positives (TP) Versus Flagged Nonconformities (FNC)

Table 2: $\quad$ Conformance Testing Outcomes 8

$\begin{array}{ll}\text { Table 3: } \quad \text { Nominal Limiting Quality } & 14\end{array}$

Table 4: $\quad$ Failure Mode, Effects, and Criticality Analysis 16

Table 5: Attribute Agreement Analysis Test Results 23

Table 6: $\quad$ Flagged Nonconformities, System A 32

Table 7: $\quad$ Analysis Results, System A 33

Table 8: $\quad$ Flagged Nonconformities, System B $\quad 34$

Table 9: $\quad$ Analysis Results, System B $\quad 35$ 
CMU/SEI-2012-TN-013 | vi 


\section{Acknowledgments}

We would like to acknowledge the contributions of the following individuals to the research presented in this paper: Doug Gwyn, Paul Anderson, William Scherlis, Jonathan Aldrich, Yekaterina Tsipenyuk O’Neil, Roger Scott, David Keaton, Thomas Plum, Dean Sutherland, Timothy Morrow, Austin Montgomery, and Martin Sebor. We would also like to acknowledge the contribution of the Software Engineering Institute support staff and management, including Paul Ruggiero, Shelia Rosenthal, Terry Ireland, Sandra Brown, Michael Wright, Archie Andrews, Bill Wilson, Rich Pethia, and Clyde Chittister. This research was supported by the U.S. Department of Energy (DOE) and the U.S. Department of Defense (DoD). 
CMU/SEI-2012-TN-013 | viii 


\section{Abstract}

The Source Code Analysis Laboratory (SCALe) is a proof-of-concept demonstration that software systems can be conformance tested against secure coding standards. CERT ${ }^{\circledR}$ secure coding standards provide a detailed enumeration of coding errors that have resulted in vulnerabilities for commonly used software development languages. The SCALe team at the CERT Program, part of Carnegie Mellon University's Software Engineering Institute, analyzes a developer's source code and provides a detailed report of findings to guide the code's repair. After the developer has addressed these findings and the SCALe team determines that the product version conforms to the standard, the CERT Program issues the developer a certificate and lists the system in a registry of conforming systems. This report details the SCALe process and provides an analysis of selected software systems. 
CMU/SEI-2012-TN-013 | x 


\section{Introduction}

The Source Code Analysis Laboratory (SCALe) is a proof-of-concept demonstration that software systems can be conformance tested against secure coding standards. SCALe provides a consistent measure that can be used to assess the security of deployed software systems, specifically by determining if they are free of coding errors that lead to known vulnerabilities. This in turn reduces the risk to these systems from increasingly sophisticated hacker tools.

\subsection{Software Security}

Software vulnerability reports and reports of software exploitations continue to grow at an alarming rate, and a significant number of these reports result in technical security alerts. To address this growing threat to the government, corporations, educational institutions, and individuals, systems must be developed that are free of software vulnerabilities.

Coding errors cause the majority of software vulnerabilities. For example, $64 \%$ of the nearly 2,500 vulnerabilities in the National Vulnerability Database in 2004 were caused by programming errors [Heffley 2004].

The CERT ${ }^{\circledR}$ Program, part of Carnegie Mellon University’s Software Engineering Institute, takes a comprehensive approach to identifying and eliminating software vulnerabilities and other flaws. The CERT Program produces books and courses that foster a security mindset in developers, and it develops secure coding standards and automated analysis tools to help them code securely. Secure coding standards provide a detailed enumeration of coding errors that have caused vulnerabilities, along with their mitigations for the most commonly used software development languages. The CERT Program also works with vendors and researchers to develop analyzers that can detect violations of the secure coding standards.

Improving software security by implementing code that conforms to the CERT secure coding standards can be a significant investment for a software developer, particularly when refactoring or otherwise modernizing existing software systems [Seacord 2003]. However, a software developer does not always benefit from this investment because it is not easy to market code quality.

\subsection{SCALe}

To address these problems, the CERT Program has created SCALe, which offers conformance testing of software systems to CERT secure coding standards.

SCALe evaluates client source code using multiple analyzers, including static analysis tools, dynamic analysis tools, and fuzz testing. The CERT Program reports any deviations from secure coding standards to the client. The client may then repair and resubmit the software for reevaluation. Once the reevaluation process is completed, the CERT Program provides the client a report detailing the software's conformance or nonconformance to each secure coding rule. The SCALe process consists of the sequence of steps shown in Figure 1.

${ }^{\circledR} \quad \mathrm{CERT}^{\circledR}$ is a registered mark owned by Carnegie Mellon University. 
1. Client contacts CERT Program. The process is initiated when a client contacts the CERT Program with a request to evaluate a software system.

2. CERT Program communicates requirements. The CERT Program communicates requirements to the client, including (1) selection of secure coding standard(s) to be used, (2) a buildable version of the software to be evaluated, and (3) a build engineer.

3. Client provides buildable software. Client selects standard(s), provides a buildable version of the software to be evaluated, and identifies the build engineer, who is available to respond to build questions for the system.

4. CERT Program selects tool set. The CERT Program chooses and documents the tool set to be used and procedures for using that tool set in evaluation of the system.

5. CERT Program analyzes source code and generates conformance test report. The CERT Program evaluates the system against specified standard(s) and provides the conformance test results to the client. If the system is found to be conforming, the CERT Program issues a certificate and terminates the conformance testing process.

6. Client repairs software. Client has the opportunity to repair nonconforming code. Client sends system back to the CERT Program for final evaluation.

7. CERT Program issues conformance tests results and certificate. The CERT Program reevaluates the system using the tools and procedures used in the initial assessment. The CERT Program provides the conformance test results to the client and, if the system is found to be conforming, a certificate.

\section{Figure 1: SCALe Process Overview}

SCALe does not test for unknown code-related vulnerabilities, high-level design and architectural flaws, the code's operational environment, or the code's portability. Conformance testing is performed for a particular set of software, translated by a particular implementation, and executing in a particular execution environment [ISO/IEC 2005].

Successful conformance testing of a software system indicates that the SCALe analysis did not detect violations of rules defined by a CERT secure coding standard. Successful conformance testing does not provide any guarantees that these rules are not violated nor that the software is entirely and permanently secure. Conforming software systems can be insecure, for example, if they implement an insecure design or architecture.

Software that conforms to a secure coding standard is likely to be more secure than nonconforming or untested software systems. However, no study has yet been performed to prove or disprove this claim.

\subsection{Conformance Assessment}

SCALe applies conformance assessment in accordance with ISO/IEC 17000: "a demonstration that specified requirements relating to a product, process, system, person, or body are fulfilled” 
[ISO/IEC 2004]. Conformance assessment generally includes activities such as testing, inspection, and certification. SCALe limits the assessments to software systems implemented in standard versions of the $\mathrm{C}, \mathrm{C}++$, and Java programming languages.

Conformance assessment activities are characterized by ISO/IEC 17000 [ISO/IEC 2004] as

- first party. The supplier organization itself carries out conformance assessment to a standard, specification, or regulation-in other words, a self-assessment-known as a supplier's declaration of conformance.

- $\quad$ second party. The customer of the organization (for example, a software consumer) performs the conformance assessment.

- third party. A body that is independent of the organization providing the product and that is not a user of the product performs the conformance assessment.

Which type of conformance assessment is appropriate depends on the level of risk associated with the product or service and the customer's requirements. SCALe is a third-party assessment performed by the CERT Program or a CERT-accredited laboratory on behalf of the supplier or on behalf of the customer with supplier approval and involvement.

\subsection{CERT Secure Coding Standards}

SCALe assesses conformance of software systems to a CERT secure coding standard. As of yearend 2011, the CERT Program has completed two secure coding standards and has two additional coding standards under development.

The CERT C Secure Coding Standard, Version 1.0, is the official version of the C language standards against which conformance testing is performed and is available as a book from Addison-Wesley [Seacord 2008]. It was developed specifically for versions of the C programming language defined by

- ISO/IEC 9899:1999 Programming Languages - C, Second Edition [ISO/IEC 2005]

- Technical Corrigenda TC1, TC2, and TC3

- ISO/IEC TR 24731-1 Extensions to the C Library, Part I: Bounds-checking interfaces [ISO/IEC 2007]

- ISO/IEC TR 24731-2 Extensions to the C Library, Part II: Dynamic Allocation Functions [ISO/IEC 2010a]

Most of the rules in The CERT C Secure Coding Standard, Version 1.0, can be applied to earlier versions of the $\mathrm{C}$ programming language and to $\mathrm{C}++$ language programs. While programs written in these programming languages may conform to this standard, they may be deficient in other ways that are not evaluated by this conformance test.

It is also possible that maintenance releases of The CERT C Secure Coding Standard will address deficiencies in Version 1.0, and that software systems can be assessed against these releases of the standard.

The CERT Oracle Secure Coding Standard for Java includes rules and recommended practices for secure programming for Java Platform Standard Edition 6 and Java SE 7 [Long 2012]. 
There are also several CERT secure coding standards under development that are not yet available for conformance testing, including

- $\quad$ The CERT C Secure Coding Standard, Version 2.0 [CERT 2010a]

- $\quad$ The CERT C++ Secure Coding Standard [CERT 2010b]

\subsection{Automated Analysis Tools}

Secure coding standards alone are inadequate to ensure secure software development because they may not be consistently and correctly applied. Manual security code audits can be supplemented by automated analysis tools, including static analysis tools, dynamic analysis tools, tools within a compiler suite, and various testing techniques.

\subsubsection{Static Analysis Tools}

Static analysis tools operate on source code, producing diagnostic warnings of potential errors or unexpected run-time behavior. Static analysis is one function performed by a compiler. Compilers can frequently produce higher-fidelity diagnostics than analyzer tools, which can be used in multiple environments, because they have detailed knowledge of the target execution environment.

There are, however, many problems and limitations with source code analysis. Static analysis techniques, while effective, are prone to both false positives and false negatives. For example, a recent study found that more than $40 \%$ of the 210 test cases went undiagnosed by all five of the study's C and C++ source analysis tools, while only $7.2 \%$ of the test cases were successfully diagnosed by all five tools (see Figure 2) [Landwehr 2008]. The same study showed that $39.7 \%$ of 177 test cases went undiagnosed by all six of the study's Java code analysis tools and that $0 \%$ of the test cases were discovered by all six tools. Dynamic analysis tools, while producing lower rates of false positives, are prone to false negatives along untested code paths. The NIST Static Analysis Tool Exposition (SATE) also demonstrated that developing comprehensive analysis criteria for static analysis tools is problematic because there are many different perspectives on what constitutes a true or false positive [Okun 2009]. 

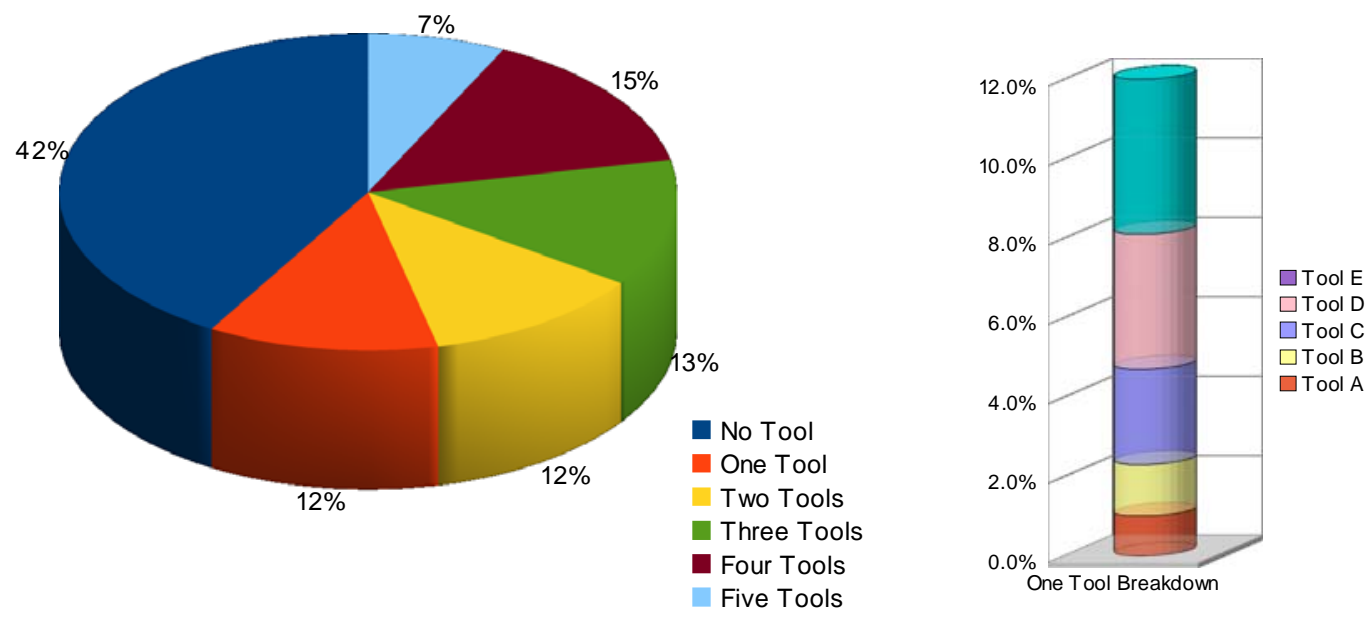

Figure 2: C and C++ "Breadth" Case Coverage [Landwehr 2008]

To address these problems, the CERT Program is working with analyzer vendors and with the WG14 C Secure Coding Guidelines Rules Group (CSCR SG) to precisely define a set of analyzable secure coding guidelines for the C99 version of the C Standard [ISO/IEC 2005], as well as for the emerging C11 major revision to the C standard [ISO/IEC 2011]. Having such a set of guidelines and standardizing them through the ISO/IEC process should eliminate many of the problems encountered at the NIST SATE and also increase the percentage of defects found by more than one tool. The CERT Program is working on tools to support the set of analyzable secure coding guidelines. First, the CERT Program is coordinating a test suite, under a Berkeley Software Distribution (BSD)-type license, ${ }^{1}$ that will be freely available for any use. This test suite can be used to determine which tools are capable of enforcing which guidelines and to establish false positive and false negative rates. Second, the CERT Program has extended the Compass/ROSE tool, ${ }^{2}$ developed at Lawrence Livermore National Laboratory, to diagnose violations of the CERT Secure Coding Standards in C and C++.

\subsubsection{Dynamic Analysis and Fuzz Testing}

Dynamic program analysis analyzes computer software by executing that software on a real or virtual processor. For dynamic program analysis to be effective, the target program must be executed with test inputs sufficient to produce interesting behavior. Software testing techniques such as fuzz testing can stress test the code [Takanen 2008], and code coverage tools can determine how many program statements have been executed.

\subsection{Portability and Security}

Portability and security are separate, and sometimes conflicting, software qualities. Security can be considered a measure of fitness for use of a given software system in a particular operating

\footnotetext{
$1 \quad$ http://www.opensource.org/licenses/bsd-license.php

2 http://www.rosecompiler.org/compass.pdf
} 
environment, as noted in Section 1.2. Software can be secure for one implementation and insecure for another. ${ }^{3}$

Portability is a measure of the ease with which a system or component can be transferred from one hardware or software environment to another [IEEE Std 610.12 1990]. Portability can conflict with security, for example, in the development of application programming interfaces (APIs) that provide an abstract layer over nonportable APIs while cloaking underlying security capabilities. Portability can become a security issue when developers create code based upon a set of assumptions for one implementation and port it, without adequate verification, to a second implementation where these assumptions are no longer valid. For example, the $\mathrm{C}$ language standard defines a strictly conforming program as one that uses only those features of the language and library specified in the standard [ISO/IEC 2005]. Strictly conforming programs are intended to be maximally portable among conforming implementations. Conforming programs may depend upon nonportable features of a conforming implementation.

Software developers frequently make assumptions about the range of target operating environments for the software being analyzed:

- The null pointer is bitwise zero. This assumption means that initializing memory with allbits-zero (such as with calloc) initializes all pointers to the null pointer value.

- A floating-point value with all bits zero represents a zero floating-point value. This assumption means that initializing memory with all-bits-zero (such as with calloc) initializes all floating-point objects to a zero value.

- A pointer-to-function can be converted to a pointer-to-void and back to a pointer-to-function without changing the value. This is true of all POSIX systems.

- Integers have a twos-complement representation. This assumption means that the bitwise operators produce well-defined results upon signed or unsigned integers, subject to restrictions upon the range of values produced.

- Integers are available for 8-, 16-, 32-, and 64-bit values. This assumption means that the library provides standardized type definitions for int 8 _t, int $16 \_t$, int $32_{-} t$, and int64_t.

While not guaranteed by the $\mathrm{C}$ standard, these assumptions are frequently true for most implementations and allow for the development of smaller, faster, and less complex software. The CERT C Secure Coding Standard encourages the use of a static assertion to validate that these assumptions hold true for a given implementation (see guideline "DCL03-C. Use a static assertion to test the value of a constant expression”) [Seacord 2008].

Because most code is constructed with these portability assumptions, it is generally counterproductive to diagnose code constructs that do not strictly conform. This would produce extensive diagnostic warnings in most code bases, and these flagged nonconformities would largely be perceived as false positives by developers who have made assumptions about the range of target platforms for the software.

An implementation is "a particular set of software, running in a particular translation environment under particular control options, that performs translation of programs for, and supports execution of functions in, a particular execution environment" [ISO/IEC 2005]. 
Consequently, conformance testing for the CERT C Secure Coding Standard is performed with respect to one or more specific implementations. A certificate is generated for the product version, but each separate target implementation increases the cost of conformance testing. It is incumbent upon the developer requesting validation to provide the appropriate bindings for implementationdefined and unspecified behaviors evaluated during conformance testing.

\subsection{SCALe for Varying Application Domains}

Because of the flexibility of the $\mathrm{C}$ language, software developed for different application domains often has significantly different characteristics. For example, applications developed for the desktop may be significantly different than applications developed for embedded systems.

For example, one of the CERT C Secure Coding Standard rules is "ARR01-C. Do not apply the si zeof operator to a pointer when taking the size of an array." Applying the si zeof operator to an expression of pointer type can result in under allocation, partial initialization, partial copying, or other logical incompleteness or inconsistency if, as is usually the case, the programmer means to determine the size of an actual object. If the mistake occurs in an allocation, then subsequent operations on the under-allocated object may lead to buffer overflows. Violations of this rule are frequently, but not always, a coding error and software vulnerability. Table 1 illustrates the ratio of true positives (bugs) to flagged nonconformities in four open source packages.

Table 1: True Positives (TP) Versus Flagged Nonconformities (FNC)

\begin{tabular}{|l|l|l|}
\hline Software System & TP/FNC & Ratio \\
\hline Mozilla Firefox version 2.0 & $6 / 12$ & $50 \%$ \\
\hline Linux kernel version 2.6.15 & $10 / 126$ & $8 \%$ \\
\hline Wine version 0.9.55 & $37 / 126$ & $29 \%$ \\
\hline xc, version unknown & $4 / 7$ & $57 \%$ \\
\hline
\end{tabular}

The ratio of true positives to flagged nonconformities shows that this checker is inappropriately tuned for analysis of the Linux kernel, which has anomalous results. Customizing SCALe to work with software for a particular application domain will help eliminate false positives in the analysis of such code, decrease the time required to perform conformance testing, and subsequently decrease the associated costs. 


\section{SCALe Process}

This section describes the processes implemented in SCALe for conformance testing against a secure coding standard.

\subsection{Conformance Testing Outcomes}

Software systems can be evaluated against one or more secure coding standards. Portions of a software system implemented in languages for which a coding standard is defined and for which conformance tests are available can be evaluated for conformance to those standards. For example, a software system that is partially implemented in PL/SQL, C, and C\# can be tested for conformance against The CERT C Secure Coding Standard. The certificate issued will identify the programming language composition of the system and note that the PL/SQL and C\# components are not covered by the conformance test.

For each secure coding standard, the source code is found to be provably nonconforming, conforming, or provably conforming against each guideline in the standard as shown in Table 2 .

Table 2: Conformance Testing Outcomes

\begin{tabular}{l|l}
$\begin{array}{l}\text { Provably } \\
\text { nonconforming }\end{array}$ & $\begin{array}{l}\text { The code is provably nonconforming if one or more violations of a rule are } \\
\text { discovered for which no deviation has been allowed. }\end{array}$ \\
\hline $\begin{array}{l}\text { Conforming } \\
\text { Provably }\end{array}$ & The code is conforming if no violations of a rule can be identified. \\
conforming & $\begin{array}{l}\text { The code is provably conforming if the code has been verified to adhere to the } \\
\text { rule in all possible cases. }\end{array}$
\end{tabular}

Strict adherence to all rules is unlikely, and, consequently, deviations associated with specific rule violations are necessary. Deviations can be used in cases where a true positive finding is uncontested as a rule violation, but the code is nonetheless determined to be secure. This may be the result of a design or architecture feature of the software or because the particular violation occurs for a valid reason that was unanticipated by the secure coding standard. In this respect, the deviation procedure allows for the possibility that secure coding rules are overly strict. Deviations will not be approved for reasons of performance, usability, or to achieve other nonsecurity attributes in the system. A software system that successfully passes conformance testing must not present known vulnerabilities resulting from coding errors.

Deviation requests are evaluated by the lead assessor, and if the developer can provide sufficient evidence that deviation will not result in a vulnerability, the deviation request will be accepted. Deviations should be used infrequently because it is almost always easier to fix a coding error than it is to provide an argument that the coding error does not result in vulnerability.

Once the evaluation process has been completed, the CERT Program delivers to the client a report detailing the conformance or nonconformance of the code to the corresponding rules in the secure coding standard. 


\subsection{SCALe Laboratory Environment}

Figure 3 shows the SCALe laboratory environment established at the CERT Program.
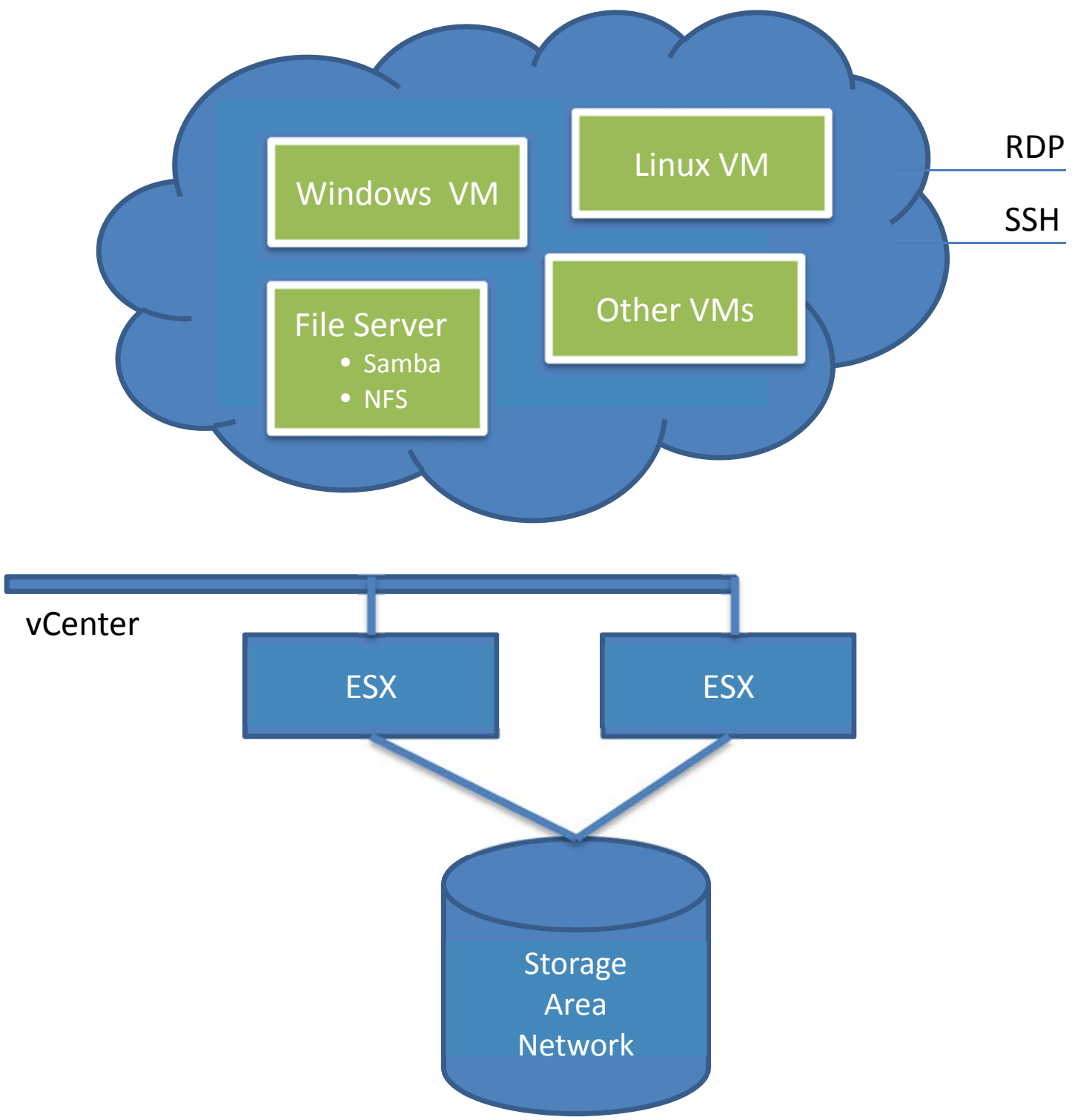

Figure 3: Source Code Analysis Laboratory

The SCALe laboratory environment consists of two servers running VMware ESX hypervisors. These are supported by a large storage area network (SAN) with redundant storage and backup capabilities. The two ESX servers support a collection of virtual machines (VMs) that can be configured to support analysis in various environments, such as Windows XP and Linux. A VMware vCenter Server provides control over the virtual environment.

The VMs are connected by a segmented-off network and to a file server running Samba and NFS. The Windows VMs can be remotely accessed from within the CERT network by using Remote Desktop Protocol (RDP) and the Linux VMs by using Secure Shell (SSH). The machines are otherwise disconnected from the internet. 
Source code being analyzed is copied onto the file server, where it is available to all the analysis VMs. Analyzers and other tools are installed through a similar process or by using vCenter.

\subsection{Conformance Testing Process}

Figure 4 illustrates the SCALe conformance testing process. The client provides the software containing the code for analysis. This software must build properly in its build environment, such as Microsoft Windows/Visual Studio or Linux/GCC. It may produce compiler warnings but may not produce fatal errors. If the target operational environment is different than the build environment, the target environment must be fully specified, including all implementation-defined behaviors.

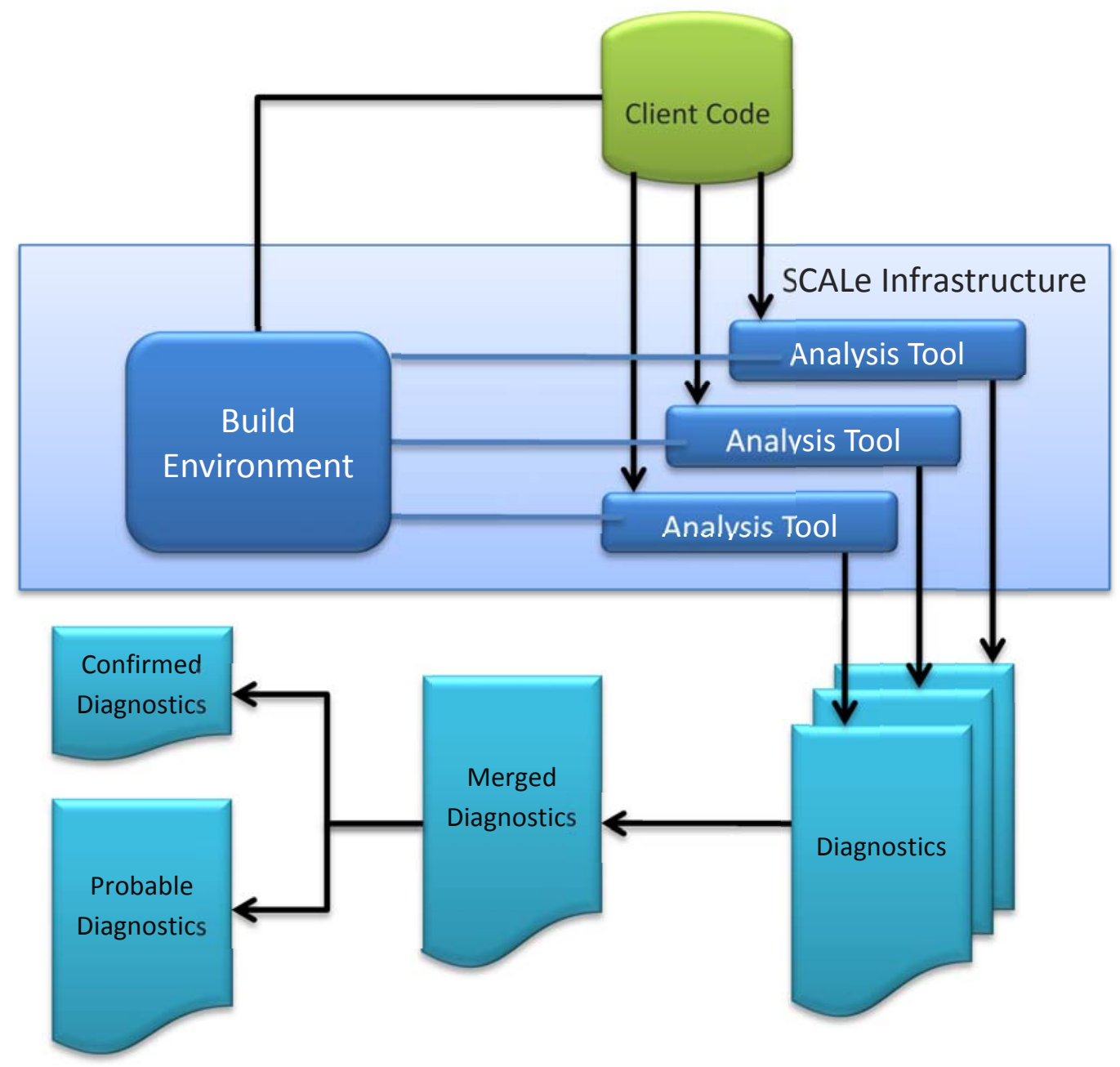

Figure 4: SCALe Conformance Testing Process 


\subsection{The Use of Analyzers in Conformance Testing}

The client code is analyzed using multiple analyzers. ${ }^{4}$ Section 1.5 contains additional background information on various types of analysis. Each analyzer accepts the client code as input and produces a set of flagged nonconformities.

Dynamic analysis tools must be able to run the program, which requires not only the correct execution environment but also suitable representative inputs. Additionally, the execution environment may include custom or special-purpose hardware, test rigs, and other equipment. These hurdles can make dynamic analysis tools challenging. The final report provided to the client documents the degree to which dynamic analysis was applied during conformance testing.

Also, an analyst may manually review the source code, using both structured and unstructured techniques, and record any violations of secure coding rules discovered. However, manual code scanning costs considerably more than automated analysis, and the results depend more on the skill and tenacity of the analyst.

Each analyzer produces a set of flagged nonconformities. Diagnostic formats vary with each tool, but they typically include the following information:

- $\quad$ name of source file where the flagged nonconformity occurs

- $\quad$ flagged nonconformity line number

- $\quad$ flagged nonconformity message (error description)

Some diagnostic messages may indicate a violation of a secure coding guideline or security violation, and others may not. Analyzer diagnostic warnings that represent violations of secure coding guidelines are mapped to the respective guideline, typically using a regular expression. This mapping can be performed directly by the tool or by the SCALe infrastructure. Analyzers that directly support a mapping to the CERT secure coding standards include Compass/ROSE, LDRA Testbed, ${ }^{5}$ and Klocwork. ${ }^{6}$

When possible, SCALe also uses dynamic analysis and fuzz testing techniques to identify coding defects and for true/false positive analysis in addition to the routinely performed static analysis. An example of this is the basic fuzzing framework (BFF) developed by the CERT Program. The BFF has two main parts:

- a Linux VM that has been optimized for fuzzing

- $\quad$ a set of scripts and a configuration file that orchestrate the fuzzing run

The VM is a stripped-down Debian installation with the following modifications:

- The Fluxbox window manager is used instead of the heavy Gnome or KDE desktop environments.

$4 \quad$ The $\mathrm{C}$ Secure Coding Rules Study Group defines an analyzer to be the mechanism that diagnoses coding flaws in software programs. This may include static analysis tools, tools within a compiler suite, and code reviewers.

5 http://www.Idra.com/certc.asp

6 http://www.klocwork.com/solutions/security-coding-standards/ 
- Fluxbox is configured not to raise or focus new windows. This can help in situations where you may need to interact with the guest operating system (OS) while a graphical user interface (GUI) application is being fuzzed.

- Memory randomization is disabled for reproducibility.

- VMware Tools is installed, which allows the guest OS to share a directory with the host.

- The OS is configured to automatically log in and start X.

- The sudo command is configured not to prompt for a password.

- The strip command is symlinked to / bin/true, which prevents symbols from being removed when an application is built.

The goal of fuzzing is to generate malformed input that causes the target application to crash. The fuzzer used by the BFF is Sam Hocevar's zzuf application. ${ }^{7}$ The CERT Program chose zzuf for its deterministic behavior, number of features, and lightweight size. By invoking zzuf from a script (zzuf.pl), additional aspects of a fuzzing run are automatable:

- $\quad$ Collect program stderr output, Valgrind memcheck, and gdb backtrace. This information can help a developer determine the cause of a crash.

- De-duplication of crashing test cases. Using gdb backtrace output, zzuf .pl determines if a crash has been encountered before. By default, duplicate crashes are discarded.

- Minimal test case generation. When a mutation causes a crash, the BFF will generate a test case where the number of bytes that are different from the seed file is minimized. By providing a minimal test case, the BFF simplifies the process of determining the cause of a crash.

The zzuf . pl reads the configuration options from the zzuf. cfg file. This file contains all of the parameters relevant to the current fuzz run, such as the target program and syntax, the seed file to be mutated, and how long the target application should be allowed to run per execution. The configuration file is copied to the guest OS when a fuzzing run has started. The zzuf script periodically saves its current progress within a fuzzing run as well. These two features work together to allow the fuzzing VM to be rebooted at any point, allowing the VM to resume fuzzing at the last stop point. The fuzzing script also periodically touches the / tmp/fuzzing file. A Linux software watchdog checks for the age of this file, and if it is older than the specified amount of time, the VM is automatically rebooted. Because some strange things can happen during a fuzzing run, this robustness is necessary for full automation. The z zuf .pl script takes this one step further by collecting additional information about the crashes. Cases that are determined to be unique are saved.

In addition to the BFF, the CERT Program has developed a GNU Compiler Collection (GCC) prototype of the as-if infinitely ranged integer (AIR) model that, when combined with fuzz testing, can be used to discover integer overflow and truncation vulnerabilities. Assuming that the source code base can be compiled with an experimental version of the GCC 4.5.0 compiler, it may be possible to instrument the executable using AIR integers. AIR integers either produce a value equivalent to that obtained using infinitely ranged integers or cause a runtime-constraint violation. Instrumented fuzz testing of libraries that have been compiled using a prototype AIR integer

http://caca.zoy.org/wiki/zzuf 
compiler has been effective in discovering vulnerabilities in software and has low false positive and false negative rates [Dannenberg 2010].

With static tools, the entire code base is available for analysis. AIR integers, on the other hand, can only report constraint violations if a code path is taken during program execution and the input data causes a constraint violation to occur.

\subsection{Conformance Test Results}

The CERT Program provides conformance test results to the client following step 5, "CERT Program analyzes source code and generates conformance test report," as shown in the SCALe process overview in Figure 1, and again following step 7, "CERT Program issues conformance tests results and certificate.”

When available, violations that do not prevent successful conformance testing, or other diagnostic information, can be provided to the client for informational purposes.

\subsubsection{Conformance Test Results Generation}

The SCALe lead assessor integrates flagged nonconformities from multiple analyzers into a single diagnostic list. Flagged nonconformities that reference the same rule violation, file, and line number are grouped together and assigned to the same analysts based on the probability that these are multiple reports of the same error. In case these do refer to different errors, the individual reports are maintained for independent analysis. However, it still makes sense to assign these as a group because the locality makes it easier to analyze them together.

Diagnostic warnings may sometimes identify errors not associated with any existing secure coding rule. This can occur for three reasons. First, it is possible that a diagnostic represents a vulnerability not addressed by any existing secure coding rule. This may represent a gap in the secure coding standard, which necessitates the addition of a new secure coding guideline. Second, a diagnostic may have no corresponding secure coding rule because the diagnostic does not represent a security flaw. Many analysis tools report portability or performance issues that are not considered to be secure coding rule violations. Third and finally, the diagnostic may be a false positive, that is, a diagnostic for which it is determined that the code does not violate a rule. False positives may arise through the normal operation of an analyzer, for example, because of the failure of a heuristic test. Alternatively, they may represent a defect in the analysis tool and consequently an opportunity to improve it. It is important to remember, however, that simultaneously avoiding both false positives and false negatives is generally impossible. Once a flagged nonconformity is determined to be a false positive, it is not considered or analyzed further.

Finally, the merged flagged nonconformities must be evaluated by a SCALe analyst to ascertain whether they are true or false positives. This is the most effort-intensive step in the SCALe process because there may be thousands of flagged nonconformities for a small- to medium-sized code base. Inspecting each flagged nonconformity is cost-prohibitive and unnecessary because it is possible to be confident - with a specified level of risk - that no true positives escape detection through statistical sampling and analysis. 
Homogeneous buckets group flagged nonconformities based on the specific analyzer checker that reported it (as determined by examining the diagnostic). A statistical sampling approach selects a random sample of flagged nonconformities from a given bucket for further investigation. The specific statistical sampling approach used is called lot tolerance percent defective (LTPD) single sampling [Stephens 2001]. This LTPD reference uses an industry standard consumer risk of $10 \%$, meaning that there is only a $10 \%$ chance of the security analyst being wrong in declaring a bucket of flagged nonconformities free of true positives based on the selected nominal limiting quality (defined in Table 3). The LTPD decision tables guiding the sample size for a given bucket require the following parameters as inputs:

1. bucket size - the number of flagged nonconformities for a given analyzer checker from which a sample will be investigated

2. nominal limiting quality (LQ) - the minimum percentage of true positives within a bucket of flagged nonconformities that the sampling plan will detect with $90 \%$ confidence and consequently confirm a violation of the coding rules

For the purposes of SCALe, the nominal LQ is assumed to be $2 \%$. Note that the higher the LQ percentage, the smaller the sample of nonconformities for further investigation.

The above parameters, when used in conjunction with published LTPD tables, will determine the required sample size $(n)$, from a bucket of flagged non-conformities associated with a given analyzer checker, that must be investigated by the SCALe analyst. Table 3 presents the set of the most likely scenarios that will be encountered by the security analysts, as derived from The Handbook of Applied Acceptance Sampling [Stephens 2001]. ${ }^{8}$ The column headings contain the nominal LQ in percent, the row headings represent the bucket size, and their intersections in the table body are the sample size required by the nominal LQ and bucket size.

Table 3: Nominal Limiting Quality

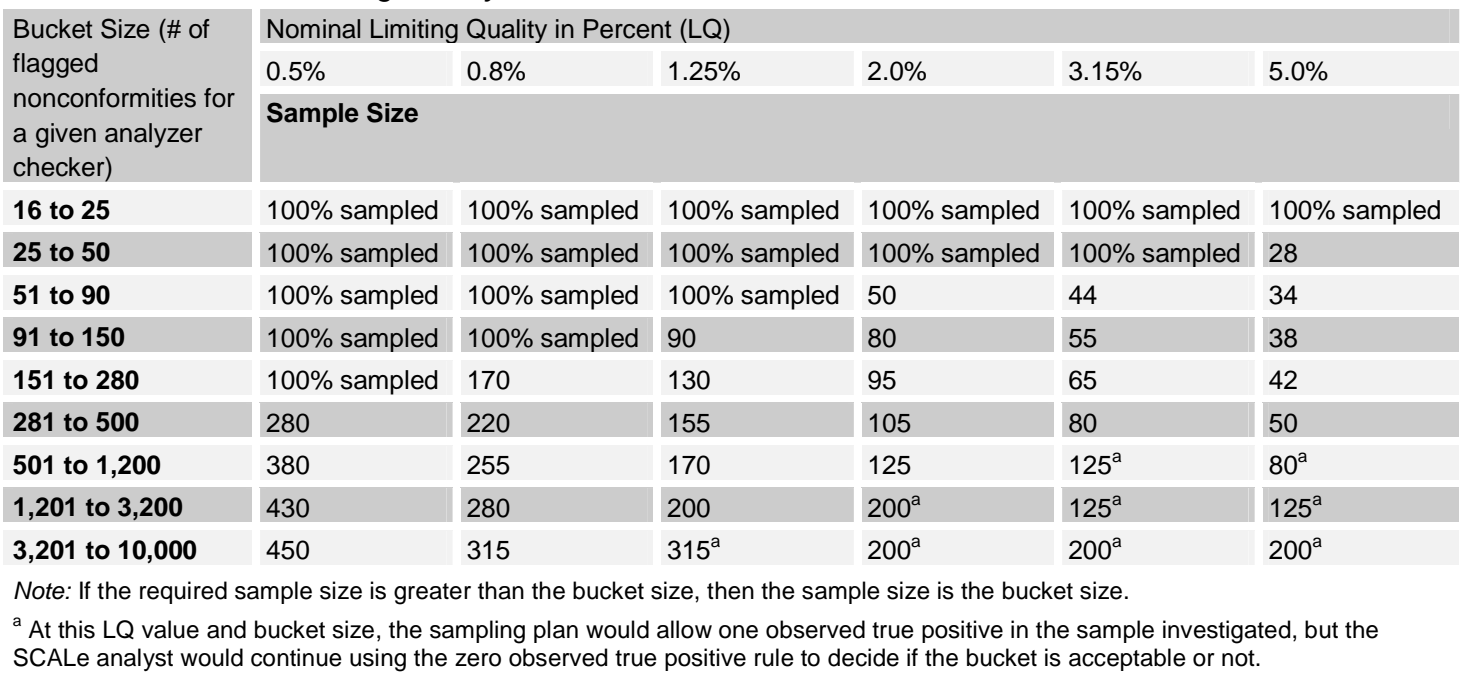

Assuming there are zero true positives found in a sample, the security analyst will be able to declare, for example, "Based on an investigation of a random sample of flagged nonconformities

For purposes of SCALe, the allowable number of defects found in a sample for a given quality level (Ac) is constrained to zero with the implication that any true positive found in a sample will be a basis for rejecting the bucket and declaring a violation of the security rule. 
within a given bucket (of an analyzer checker), there is $90 \%$ confidence that the bucket of flagged nonconformities for a given analyzer checker contains no more than $2 \%$ true positives," where $2 \%$ true positives is the previously determined nominal LQ.

The procedure consists of the following steps for each bucket:

1. Identify the nominal LQ desired for the security analysis. For example, a 5\% nominal LQ implies that the sampling scheme will identify buckets that have $5 \%$ or more true positives. The available tables offer LQ percentages of $0.5 \%, 0.8 \%, 1.25 \%, 2.0 \%, 3.15 \%, 5.0 \%$, and higher. The default LQ value for SCALe is $2 \%$.

2. Identify the bucket size (number of flagged nonconformities within a bucket for a given analyzer checker).

3. Use the table to identify the required sample size $(n)$. Note that at the $2 \%$ LQ, all flagged nonconformities are investigated if the bucket size totals 50 or fewer.

4. Randomly select the specified number $(n)$ of flagged nonconformities from the bucket.

5. Investigate each flagged nonconformity in the sample to determine whether it is a false or true positive flagged nonconformity, and label it accordingly.

6. If all flagged nonconformities in the sample are false positives, all remaining flagged nonconformities in the bucket are discarded as false positives.

7. If a flagged nonconformity in the sample is determined to be a violation of the secure coding rule, it is categorized as a confirmed violation. No further investigation is conducted of the remaining nonconformities in the bucket, and these will continue to be categorized as unknown.

At the end of this process, there may be a small set of confirmed violations and a larger set of unknown or unevaluated violations. A confirmed violation represents a genuine security flaw in the software being tested and will result in the software being found provably nonconforming with respect to the secure coding guideline and failing to pass conformance testing. The CERT Program will provide a list of unknown violations of the same secure coding rules to the client along with confirmed violations. The final diagnostic report consists of the confirmed violations together with the list of unknown violations.

\subsubsection{Additional Documentation}

Each rule provides additional information, including a description of the rule, noncompliant code examples, compliant solutions, and risk assessment, that provides software developers with an indication of the potential consequences of not addressing a particular vulnerability in their code (along with some indication of expected remediation costs). This metric is based on failure mode, effects, and criticality analysis (FMECA) [IEC 2006]. A development team can use this information to prioritize the repair of vulnerability classes. ${ }^{9}$ It is generally assumed that new code will be developed to be compliant with all applicable guidelines.

As seen in Table 4, each rule in the CERT C Secure Coding Standard is scored on a scale of 1 to 3 for severity, likelihood, and remediation cost.

$9 \quad$ Vulnerability metrics, such as the Common Vulnerability Scoring System (CVSS), measure the characteristics and impacts of specific IT vulnerabilities, not the risk from a coding rule violation. 
Table 4: Failure Mode, Effects, and Criticality Analysis

\begin{tabular}{|c|c|c|c|c|}
\hline \multirow{3}{*}{$\begin{array}{l}\text { Severity - How serious are } \\
\text { the consequences of the rule } \\
\text { being ignored? }\end{array}$} & Value & Meaning & \multicolumn{2}{|c|}{ Examples of Vulnerability } \\
\hline & 1 & low & \multicolumn{2}{|c|}{ denial-of-service attack, abnormal termination } \\
\hline & 2 & medium & \multicolumn{2}{|c|}{$\begin{array}{l}\text { data integrity violation, unintentional information } \\
\text { disclosure }\end{array}$} \\
\hline \multirow{5}{*}{$\begin{array}{l}\text { Likelihood - How likely is it } \\
\text { that a flaw introduced by } \\
\text { ignoring the rule can lead to an } \\
\text { exploitable vulnerability? }\end{array}$} & 3 & high & \multicolumn{2}{|c|}{ run arbitrary code } \\
\hline & Value & Meaning & \\
\hline & 1 & unlikely & & \\
\hline & 2 & probable & & \\
\hline & 3 & likely & & \\
\hline \multirow{4}{*}{$\begin{array}{l}\text { Cost - How much will } \\
\text { mitigating the vulnerability } \\
\text { cost? }\end{array}$} & Value & Meaning & Detection & Correction \\
\hline & 1 & high & manual & manual \\
\hline & 2 & medium & automatic & manual \\
\hline & 3 & low & automatic & automatic \\
\hline
\end{tabular}

\subsection{Tracking Diagnostics Across Code Base Version}

Infrequently, source code submitted for conformance assessment will be discovered to be free from secure coding violations on the initial assessment. More commonly, at least a single iteration is required. Consequently, this iteration has been designed into the process. Often, multiple iterations are required to discover and eliminate secure coding violations in software that has not been developed in conformance with the appropriate secure coding standards.

Depending on the analyzers used, it is not uncommon for code bases to have substantial numbers of false positives in addition to the true positives that caused the software to fail conformance testing. False positives must be eliminated before a software system can be determined to be conforming. However, analyzing the code to determine which diagnostics are false positives is time consuming and labor intensive. Furthermore, this process needs to be repeated each time the code base is submitted for analysis. Consequently, preventing the issuance of diagnostics determined to be false positives can reduce the cost and time required for conformance testing in most cases.

Diagnostics determined to be false positives can be eliminated in a variety of ways. Code constructs may be diagnosed because they correspond to common programmer errors. In other cases, these same code constructs may be intentional, but the analyzer cannot determine that a particular usage is secure. In these cases, the programmer simply needs a mechanism to express design intent more clearly.

Design intent can be expressed with the stylistic use of code or with special annotations. For example, given a guideline such as "FIO04-C. Detect and handle input and output errors," the following line of code would require a diagnostic:

$$
\text { puts(".."); / / diagnostic required }
$$

However, the following code would be considered conforming:

$$
\begin{aligned}
& \text { if }(\mathrm{EOF}==\operatorname{puts}(" \ldots l)) \quad / / \text { okay: error handled } \\
& \text { exit }(1) ;
\end{aligned}
$$


If the failure to test the return value from the puts function was intentional, this design intent could be expressed by casting the resulting expression to void:

$$
\text { (void)puts(".."); / / don't care about errors here }
$$

Special comments or pragmas may also be used for this purpose. For example, lint is silent about certain conditions if a special comment such as /*VARARGS2* / or / *NOTREACHED* / is embedded in the code pattern triggering them. The comment

$$
\text { /*NOTREACHED* / }
$$

is equivalent to

$$
\text { \#pragma notreached }
$$

Of course, to suppress the diagnostic, both approaches must be recognized by the analyzer, and there is no standard set of stylistic coding conventions (although some conventions are more widely adopted than others).

Both approaches also require modification of source code, which, of course, is not a process or output of conformance testing. In fact, diagnostics are typically unsuppressed during analysis to ensure that secure coding violations are not inadvertently being suppressed.

A related approach is frequently referred to as "stand-off annotations," in which the annotations are external to the source code. This approach is more practical for SCALe and other processes in which the source code cannot be modified.

In step 6 of the SCALe process overview shown in Figure 1, the client has the opportunity to repair nonconforming code and can send the system back to the CERT Program for a further assessment. Because the initial and subsequent code bases are separated by time and potentially multiple code restructurings, it can be difficult to match a new flagged nonconformity with a flagged nonconformity from an earlier version of the system. No matching technique will be perfect for all users, and it may fail in two ways:

1. It may fail to match a flagged nonconformity that should have been matched, so the false positive reappears.

2. It may erroneously match a flagged nonconformity that should have been treated separately. In this case the old flagged nonconformity's annotation will replace the newer flagged nonconformity. If the old flagged nonconformity was annotated as a false positive and the new flagged nonconformity is a true positive, then the user may never see it, creating a false negative.

GrammaTech CodeSonar, Coverity Prevent, and Fortify Source Code Analysis (SCA) each have a proprietary solution for solving this problem. SCALe could use these proprietary mechanisms to indicate at the individual tool level which diagnostics are false positives and should no longer be reported. This solution may be effective, but it requires direct access to the tool (as opposed to dealing strictly with aggregate results), and this approach is only feasible when the underlying tool provides the mechanism. Another drawback is that the false positive must be silenced by the conformance tester in each reporting analyzer. 
Following the initial generation of a diagnostic report as described in Section 2.5.1, each diagnostic also has a validity status: true, probably true, unknown, probably false, or false. Each diagnostic starts in the unknown state. Any diagnostic that is manually inspected by an auditor becomes true or false. When the audit is complete, all other diagnostics will be probably true or probably false. This information needs to be transferred from the previous conformance test to minimize the amount of time spent reevaluating false positive findings.

\subsubsection{Standard Approach}

A potentially feasible approach to standardization is to specify a \#pragma for analyzers to implement. With the _Pragma operator, the pragma name and number would not need to be the same across tools, although it would help if the pragma name and number mapped to equivalent functionalities such as those being produced by the WG14 C Secure Coding Rules Study Group. The following code illustrates a standard approach to using pragmas to suppress diagnostics:

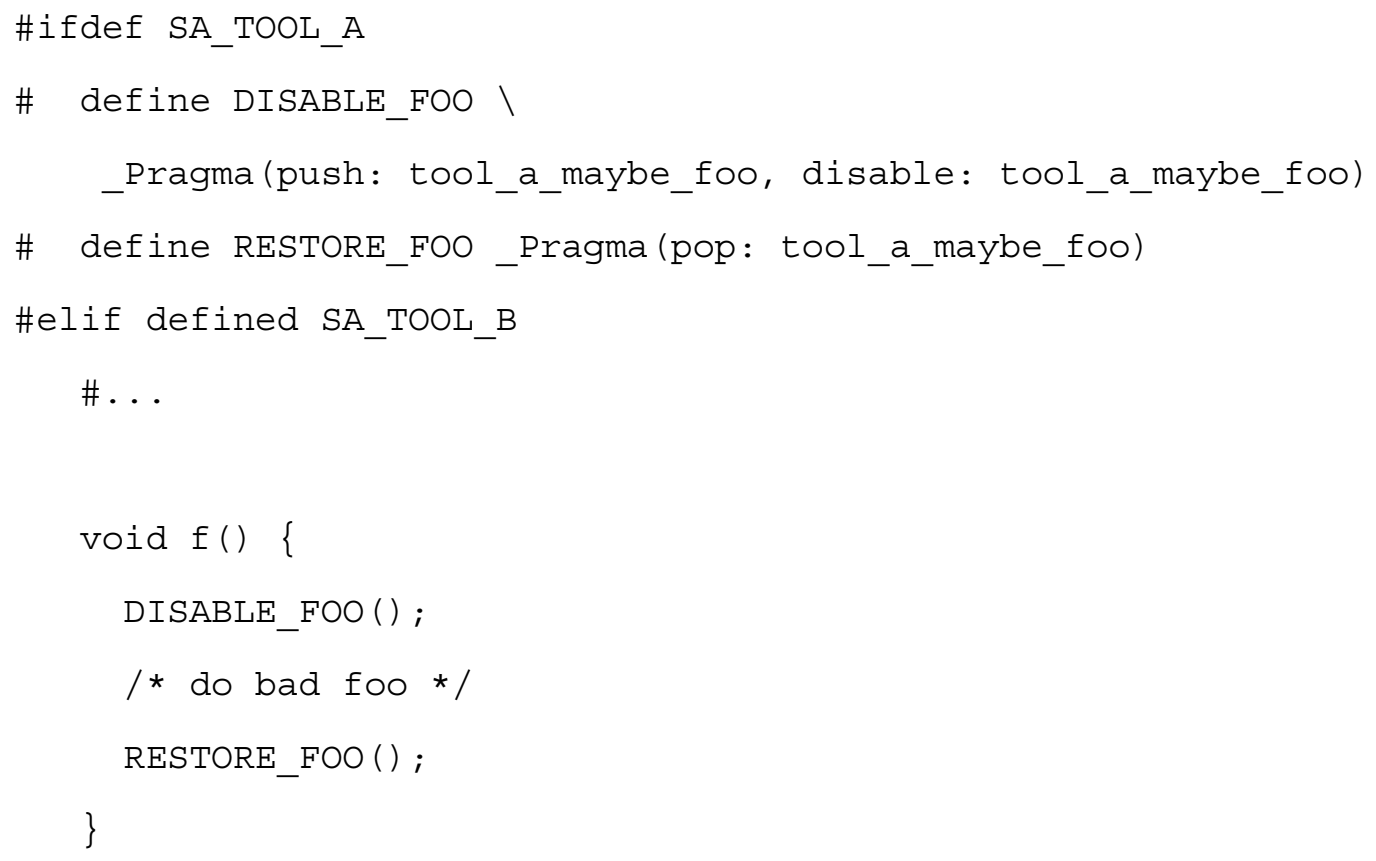

Unfortunately, there are serious practical obstacles to portability when using pragmas.

The biggest problem with pragmas is that even though the language requires implementations to ignore unknown pragmas, they tend to be diagnosed by strict compilers. Compliers that do not do so make debugging incorrect uses of otherwise recognized pragmas difficult.

Another caveat about pragmas is that they have the effect of applying to whole statements rather than to expressions. Consider this example:

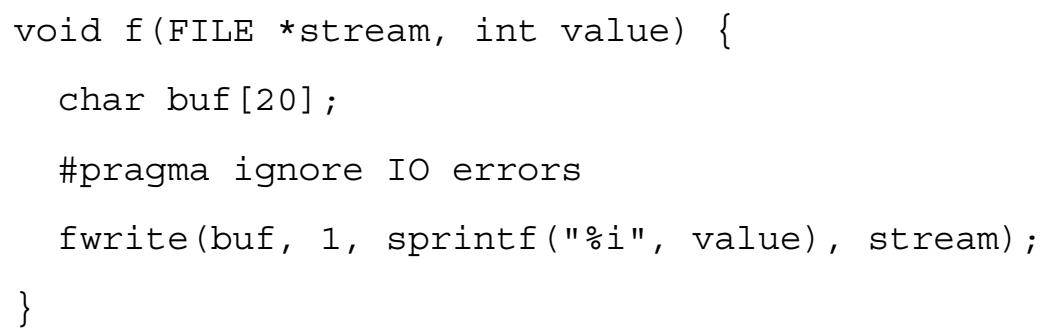


The pragma silences the diagnostics for both $\mathrm{I} / \mathrm{O}$ functions on the next line, and it is impossible to make it silence just one and not the other. Of course, it is possible to rewrite this code so that the pragma would apply only to a single function call.

Developers submitting software for analysis are not required to silence unwanted diagnostics.

\subsection{Quality Control}

\subsubsection{Personnel}

\subsubsection{Training}

All SCALe lab personnel undergo basic security training and specialized training as required. Everyone, including those with client-facing roles, must have a computer science degree or equivalent education, specific training in the application of a particular secure coding standard, and training in conformance assessment using SCALe.

Currently, conformance assessment is being performed only with the CERT C Secure Coding Standard; therefore secure coding training required for personnel is one of the following:

- $\quad$ Software Engineering Institute (SEI) Secure Coding in $C$ and $C++{ }^{10}$

- $\quad$ Carnegie Mellon University 15-392 Special Topic: Secure Programming ${ }^{11}$

- $\quad$ Carnegie Mellon University 14-735 Secure Software Engineering ${ }^{12}$

- An equivalent course determined by the CERT Program

Following completion of training, a new SCALe employee undergoes an apprenticeship with a trained SCALe staff person. Upon successful completion of the apprenticeship-where success is determined by skill and capability, not by the passage of time-the new SCALe employee may work independently. However, the new employee, and all employees of SCALe, will continue to work under the transparency and audit controls described in this section.

All SCALe staff members undergo ethics training to ensure that SCALe conforms to the requirements of the CERT Program, the SEI, and ISO/IEC 17000.

\subsubsection{Roles}

There are a number of defined roles within the SCALe lab.

- $\quad$ SCALe build specialist

- Responsibilities: Installs the customer build environment on the SCALe lab machines

- $\quad$ SCALe analyst

- Responsibilities: Evaluates flagged nonconformities to determine if they represent violations of secure coding rules.

- Additional training: Analysts must satisfactorily complete a formative evaluation assessment, as discussed in Section 2.7.2.

\footnotetext{
10 http://www.sei.cmu.edu/training/p63.cfm

$11 \mathrm{https}: / / \mathrm{ww}$.securecoding.cert.org/confluence/display/sci/15392+Secure+Programming

12 http://www.ini.cmu.edu/degrees/psv_msit/course_list.html
} 
- $\quad$ SCALe lead assessor

- Responsibilities: Organizes and supervises assessment activities, including supervising analyzers, tool selection, and drafting of reports.

- Additional training: Has performed at least three assessments as a SCALe analyst.

- $\quad$ SCALe assessment administrator

- Responsibilities: Develops and administers analyzer assessments.

- $\quad$ SCALe manager

- Responsibilities: Handles business relationships, including contracting, communications, and quality assurance.

\subsubsection{Quality Assurance Procedures}

Every point where human judgment comes into play is an opportunity for SCALe to generate results that are not reproducible. This will be mitigated. Each judgment point will have a documented process for making that judgment. Personnel will be trained to faithfully apply the processes. A system of review will be established, applied, and documented. The judgment will include at least the following.

Flagged nonconformity assessment: Much of the work of a conformity assessment is the human evaluation of the merged flagged nonconformities produced by the automated assessment tools. Different SCALe analysts each evaluate a subset of the flagged nonconformities. The intersection of those subsets is not the null set and is known only to the lead assessor. Consequently, SCALe analysts will perform audits of each other while simply doing their work. Any disagreement in results between SCALe analysts triggers a root cause assessment and corrective action.

Client qualification: Client qualification refers to the readiness of the client to engage the SCALe lab for analysis. The SCALe manager applies guidelines to determine if the potential client has the organizational maturity to provide software along with the build environment, respond to communications, maintain standards and procedures, and so forth. The tangible work products form an audit trail. The CERT Program will conduct periodic review of the audit trail.

Tool selection: Because there is great inter-tool variation in flagging nonconformities, the selection of tools can have considerable impact on results. It is critical that SCALe lab conformance testing results be repeatable regardless of which particular person is selecting the tool set. The SCALe manager specifies, applies, and audits well-defined procedures for tool selection.

Conformance Test Completion: Because there will be far more flagged nonconformities than will be evaluated by SCALe analysts, the SCALe process applies statistical methods. Determining when enough flagged nonconformities have been evaluated is a well-defined process documented in Section 2.5.1.

Report generation: Final reports will be based on a template of predetermined parts, including, but not limited to, a description of software and build environment, the client's tolerance for missing nonconformities (typically less that 10\%), tool selection, merged and evaluated flagged nonconformities, and stopping criterion. Both the SCALe lead assessor and the SCALe manager 
will sign off on each report. Each report will be reviewed by SEI communications for conformance with SEI standards.

\subsubsection{Attribute Agreement Analysis}

Attribute agreement analysis is a statistical method to determine the consistency of judgment within and between different SCALe analysts. Popular within the behavioral sciences, attribute agreement analysis remains a key method to determining agreement within and between raters, in this case SCALe analysts [von Eye 2006].

Simply, attribute agreement analysis constructs and implements a brief experiment in which the SCALe analysts participate in a short exercise of rendering judgment on a series of flagged nonconformities. The exercise specifically includes a variety of flagged nonconformities mapped to different rules. Attribute agreement analysis evaluates the judgments as correct or incorrect, based on the flagged nonconformity being a true positive or a false positive. In these situations, an attribute agreement measures the true-or-false positive judgment similarly to the traditional use of attribute agreement analysis in the quality control domain for pass/fail situations. The attribute agreement measure provides feedback in several dimensions:

- $\quad$ individual accuracy (for example, what percentage of judgments are correct)

- individual consistency (for example, how consistent is the individual in rendering the same judgment across time for the same or virtually the same flagged nonconformity; often referred to as repeatability)

- group accuracy (for example, what percentage of the time does a specific group of SCALe analysts render the correct judgment)

- group consistency (for example, what percentage of the time does a specific group of SCALe analysts render the same judgment for a given flagged nonconformity across time; often referred to as reproducibility)

Any modern statistical package can easily determine the attribute agreement measures, which are interpreted as follows [Landis 1977]:

- $\quad$ Less than 0 (no agreement)

- $\quad 0-0.20$ (slight agreement)

- $\quad 0.21-0.40$ (fair agreement)

- $\quad 0.41-0.60$ (moderate agreement)

- $\quad 0.61-0.80$ (substantial agreement)

- $\quad$ 0.81-1 (almost perfect agreement)

A need may arise to assess both accuracy and consistency of SCALe analysts' judgments with measures that extend beyond the binary situation (correct or incorrect) to situations in which a judgment is a gradual measure of closeness to the right answer. In this case, analysts should use an alternative attribute agreement measure and interpret the output quite similarly to the Kappa coefficient, with results possible on the dimensions listed above. As such, Kendall coefficients serve well for judgments on an ordinal scale, in which incorrect answers are closer or farther away from the correct answer. A hypothetical example of a judgment on an ordinal scale would be if a SCALe analyst were asked to render judgment of the severity of a flagged nonconformity, say on 
a 10-point scale. If the true severity is, for example, 8, and two SCALe analysts provided answers of 1 and 7, respectively, then a severity judgment of 7 would have a much higher Kendall coefficient than the severity judgment of 1 .

In conclusion, attribute agreement analysis may be conducted via small exercises with SCALe analysts rendering judgments on a reasonably-sized list of different types of flagged nonconformities mapped to the set of rules within the scope of a given code conformance test.

\subsubsection{Formative Evaluation Assessment Using Attribute Agreement Analysis}

SCALe analysts participate in a formative evaluation assessment as part of their training and certification. Certification of a candidate as a SCALe analyst requires attribute agreement scores of $80 \%$ or higher. In addition, acceptable thresholds for accuracy may be imposed separately for each rule.

The formative evaluation assessment implements a simple attribute agreement analysis as follows.

First, the SCALe assessment administrator identifies a preliminary set of 20 to 35 different flagged nonconformities (from the diagnostic output of a software system or code base) for the evaluation assessment. The administrator ensures that the preliminary set includes a variety of diagnostic codes mapped to a representative collection of security rules. The administrator then identifies a second set of 20 to 35 different flagged nonconformities, such that there is a similarity mapping between each flagged nonconformity in the first set to a corresponding flagged nonconformity in the second set. The resulting complete set of 40 to 70 flagged nonconformities is then randomized and used as a test instrument for the participating SCALe analysts to evaluate.

Second, different SCALe analysts are identified to participate in the evaluation assessment. Initially, there must be at least two analysts to conduct the evaluation assessment. Subsequently, additional analysts will take the same evaluation assessment using the same or a similar set of flagged nonconformities.

Third, the SCALe analysts independently evaluate each flagged nonconformity within the complete set as either a true or false positive, recognizing true positives as rule violations.

Because human judgment can vary across time (for example, SCALe analysts may fall out of practice in exercising their judgment of flagged nonconformities) and because the scope and nature of flagged nonconformities and rules may vary across time, the CERT Program retests SCALe analysts using attribute agreement analysis every three years as part of recertification.

Lastly, the SCALe manager uses the results of ongoing attribute agreement exercises to identify ways to improve the training of SCALe analysts, including possible additional job aids. SCALe analysts will also be interviewed for context information surrounding incorrect judgments as part of this improvement activity.

Thresholds will be maintained at established levels until and unless experience indicates that they should change. 


\subsubsection{Attribute Agreement Analysis Test Experiment}

To qualify potential analyst candidates, the SCALe assessment administrator conducted an attribute agreement analysis test. The test consisted of 60 flagged nonconformities divided into pairs of similar flagged nonconformities, each having the same validity.

The administrator assigned all flagged nonconformities a numeric ID to identify pairs. The administrator constructed the test (and answer key) and assigned the test to four SCALe analyst candidates. The analyst candidates had no qualifications for code analysis other than being competent programmers. Each analyst candidate made a true or a false positive determination for each flagged nonconformity. Afterward, the analyst candidates and administrator compared results. While the administrator had initially created the answer key, the group came to different conclusions about some of the diagnostics. Table 5 presents the results of the test. The column marked "AA" contains the results for the assessment administrator's answer key, while the columns marked “AC \#” are the results for the four analyst candidates tested.

Table 5: Attribute Agreement Analysis Test Results

\begin{tabular}{|c|c|c|c|c|c|c|c|}
\hline ID & Rule & Group & AA & AC 1 & AC 2 & AC 3 & AC 4 \\
\hline 1 & DCL32-C & False & False & True & False & False & False \\
\hline 1 & DCL32-C & False & False & False & True & False & False \\
\hline 2 & ООР32-СРP & True & True & True & True & True & True \\
\hline 2 & OOP32-СРP & True & True & True & True & True & True \\
\hline 3 & MEM41-CPP & False & False & True & True & False & False \\
\hline 3 & MEM41-CPP & False & False & True & True & False & False \\
\hline 4 & MEM40-СРP & False & False & False & True & False & False \\
\hline 4 & MEM40-СРP & False & False & True & True & True & False \\
\hline 5 & EXP34-C & False & False & True & False & True & False \\
\hline 5 & EXP34-C & False & False & True & False & True & False \\
\hline 6 & DCL35-C & True & False & True & False & False & True \\
\hline 6 & DCL35-C & True & False & True & True & False & True \\
\hline 7 & ERR33-CPP & False & False & False & True & False & False \\
\hline 7 & ERR33-CPP & False & False & False & True & True & False \\
\hline 8 & ERR33-CPP & True & True & False & False & False & True \\
\hline 8 & ERR33-CPP & True & True & False & True & True & True \\
\hline 9 & EXP36-C & True & False & False & True & False & True \\
\hline 9 & EXP36-C & True & False & False & True & True & True \\
\hline 10 & EXP35-CPP & True & True & True & True & True & True \\
\hline 10 & ЕXP35-СРP & True & True & True & True & False & True \\
\hline 11 & DCL36-C & False & False & True & True & True & False \\
\hline 11 & DCL36-C & False & False & True & True & True & False \\
\hline 12 & FLP36-C & False & False & False & False & False & False \\
\hline 12 & FLP36-C & False & False & False & False & False & False \\
\hline 13 & FIO30-C & False & False & False & False & False & False \\
\hline 13 & FIO30-C & False & False & False & True & True & False \\
\hline 14 & FLP34-C & False & False & True & False & False & False \\
\hline
\end{tabular}




\begin{tabular}{|c|c|c|c|c|c|c|c|}
\hline ID & Rule & Group & $\mathrm{AA}$ & AC 1 & AC 2 & AC 3 & $\mathrm{AC} 4$ \\
\hline 14 & FLP34-C & False & False & True & False & False & False \\
\hline 15 & FLP34-C & True & True & True & True & True & True \\
\hline 15 & FLP34-C & True & True & True & False & True & True \\
\hline 16 & ARR30-C & False & True & False & True & True & False \\
\hline 16 & ARR30-C & False & True & False & True & True & False \\
\hline 17 & STR38-C & True & True & True & True & True & True \\
\hline 17 & STR38-C & True & True & True & True & True & True \\
\hline 18 & ООР37-СРP & True & True & True & True & True & True \\
\hline 18 & ООР37-СРP & True & True & False & True & False & True \\
\hline 19 & OOP37-СРP & False & False & True & True & False & False \\
\hline 19 & ООР37-СРP & False & False & True & True & False & False \\
\hline 20 & DCL31-C & False & False & False & False & True & False \\
\hline 20 & DCL31-C & False & False & False & False & True & False \\
\hline 21 & DCL31-C & False & False & False & False & False & False \\
\hline 21 & DCL31-C & False & False & False & False & False & False \\
\hline 22 & INT31-C & False & False & False & False & False & False \\
\hline 22 & INT31-C & False & False & True & False & False & False \\
\hline 23 & INT31-C & False & False & False & False & False & False \\
\hline 23 & INT31-C & False & False & False & False & False & False \\
\hline 24 & INT31-C & False & False & True & True & False & False \\
\hline 24 & INT31-C & False & False & True & True & False & False \\
\hline 25 & MSC34-C & True & True & True & True & True & True \\
\hline 25 & MSC34-C & True & True & True & True & True & True \\
\hline 26 & MSC34-C & False & False & True & True & False & False \\
\hline 26 & MSC34-C & False & False & True & True & False & False \\
\hline 27 & EXP36-C & False & False & True & True & False & False \\
\hline 27 & EXP36-C & False & False & True & True & True & False \\
\hline 28 & INT35-C & True & True & True & True & True & True \\
\hline 28 & INT35-C & True & True & True & True & True & True \\
\hline 29 & EXP34-C & True & True & False & False & True & True \\
\hline 29 & EXP34-C & True & True & True & True & True & True \\
\hline 30 & MEM41-CPP & False & False & True & True & False & False \\
\hline 30 & MEM41-CPP & False & False & False & False & False & False \\
\hline
\end{tabular}

The administrator's findings correlated strongly with the group results. The administrator shared 54 of 60 answers with the group, for a score of $90 \%$. The analyst candidates' scores showed considerably lower correlation, with results of $56.7 \%$, 58.3\%, 70\%, and 75\%, respectively. This would rate analyst candidates 1 and 2 in moderate agreement with the group and analyst candidates 3 and 4 in substantial agreement.

Most analyst candidates displayed only moderate consistency. They gave the same answer to the similar flagged nonconformity pairs most of the time but not always. Analyst candidate 1 gave the same answer to similar flagged nonconformity pairs 24 out of 30 times, for a consistency score of 
83.3\%. The second and third analyst candidates gave the same answer 23 out of 30 times, for a consistency score of $76.7 \%$. The fourth analyst candidate was extremely consistent, giving the same answer 29 out of 30 times for a consistency score of $96.7 \%$.

Attribute agreement analysis can also be conducted with the Minitab 16 Statistical Software ${ }^{13}$ as shown in Figure 5. The "Within Appraisers" section depicts the degree of internal consistency for each analyst and the administrator. Only the administrator and analyst candidate 4 have acceptable Kappa values indicating very good internal consistency. All of the $p$ values are less than 0.05 , indicating that these results are statistically significant and not due to chance. The "Each Appraiser vs Standard" section depicts how accurate each analyst and the administrator are in getting the correct answer. Again, the administrator and analyst candidate 4 have acceptable Kappa values, indicating very good accuracy in determining both false and true positives. The $p$ values less than 0.05 for the administrator and analyst candidate 4 indicate that the Kappa values are statistically significant and not due to chance.

13 http://www.minitab.com/en-US/products/minitab/default.aspx 


\section{Attribute Agreement Analysis for Response}

\section{Within Appraisers}

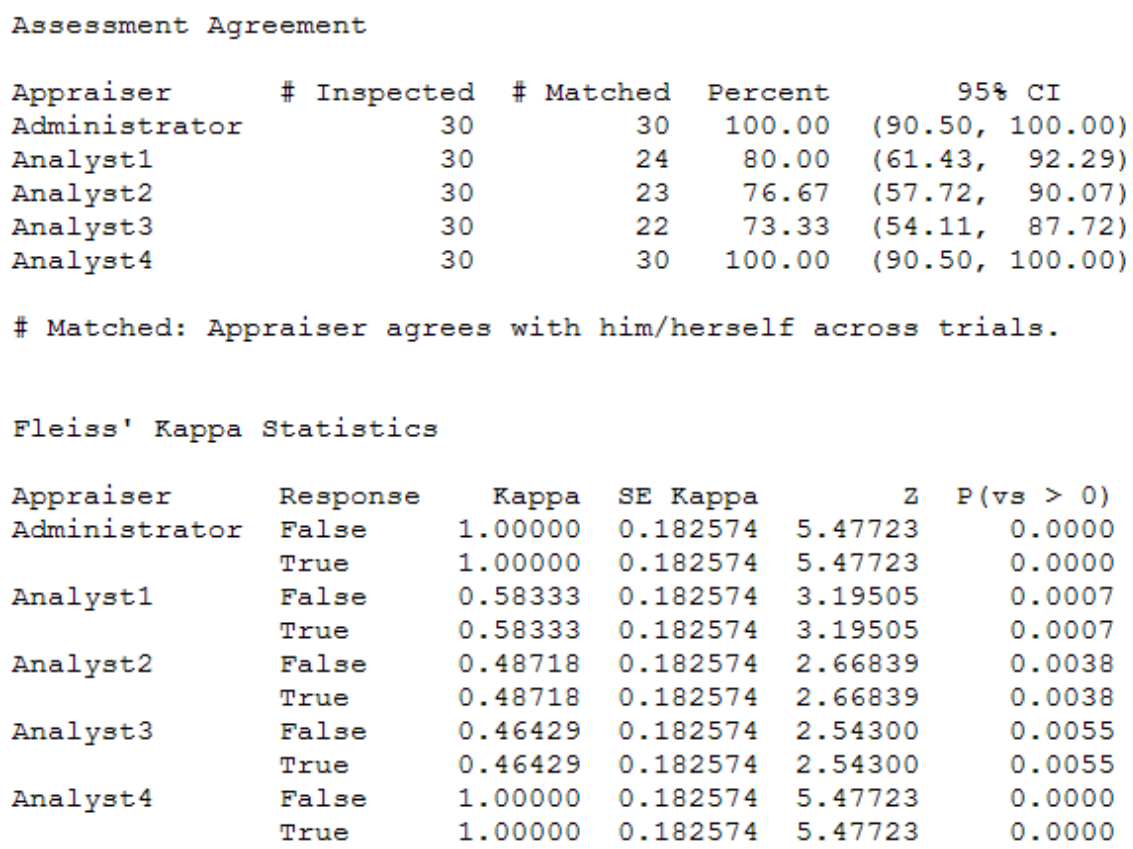

\section{Each Appraiser vs Standard}

Assessment Agreement

$\begin{array}{lrrrrr}\text { Appraiser } & \text { \# Inspected } & \text { \# Matched } & \text { Percent } & 958 \text { CI } \\ \text { Administrator } & 30 & 27 & 90.00 & (73.47, & 97.89) \\ \text { Analyst1 } & 30 & 14 & 46.67 & (28.34, & 65.67) \\ \text { Analyst2 } & 30 & 14 & 46.67 & (28.34, & 65.67) \\ \text { Analyst3 } & 30 & 17 & 56.67 & (37.43, & 74.54) \\ \text { Analyst4 } & 30 & 30 & 100.00 & (90.50,100.00)\end{array}$

\# Matched: Appraiser's assessment across trials agrees with the known standard.

$\begin{array}{llrrrr}\text { Fleiss' Kappa } & \text { Statistics } & & & \\ \text { | } & & & & \\ \text { Appraiser } & \text { Response } & \text { Kappa } & \text { SE Kappa } & \text { Z } & \text { P(vs }>0) \\ \text { Administrator } & \text { False } & 0.78022 & 0.129099 & 6.04356 & 0.0000 \\ & \text { True } & 0.78022 & 0.129099 & 6.04356 & 0.0000 \\ \text { Analyst1 } & \text { False } & 0.13237 & 0.129099 & 1.02533 & 0.1526 \\ & \text { True } & 0.13237 & 0.129099 & 1.02533 & 0.1526 \\ \text { Analyst2 } & \text { False } & 0.16440 & 0.129099 & 1.27343 & 0.1014 \\ \text { Analyst3 } & \text { True } & 0.16440 & 0.129099 & 1.27343 & 0.1014 \\ & \text { False } & 0.38286 & 0.129099 & 2.96563 & 0.0015 \\ \text { Analyst4 } & \text { True } & 0.38286 & 0.129099 & 2.96563 & 0.0015 \\ & \text { False } & 1.00000 & 0.129099 & 7.74597 & 0.0000 \\ & \text { True } & 1.00000 & 0.129099 & 7.74597 & 0.0000\end{array}$

Figure 5: Attribute Agreement Analysis Using Minitab 
The analyst candidate errors resulted from a number of factors. Many candidates, while knowledgeable in $\mathrm{C}$, possessed only a rudimentary knowledge of $\mathrm{C}++$. The analyst candidates expressed a lack of confidence with $\mathrm{C}++$. However, when ignoring the results for $\mathrm{C}++$ specific rules, there were 21 flagged nonconformity pairs, or 42 individual flagged nonconformities. For the $\mathrm{C}$ subset, the administrator shared 36 of 42 answers with the group, for a score of $85.7 \%$. The analyst candidates scored 59.5\%, 40.5\%, and 69\%, respectively. We would conclude from this that while lack of $\mathrm{C}++$ experience made the analyst candidates less confident with their test results, they did not do significantly worse on the C++-flagged nonconformities than they did with the C-flagged nonconformities.

Some errors resulted from a lack of in-depth knowledge of C. For example, two analyst candidates incorrectly confused the harmless format string " $\mathrm{d} \backslash \mathrm{n}$ ” with the more notorious format string “\% $\mathrm{n}$ ”. Ignorance of the Windows function calls and types employed by the code led to some mistakes.

Analyst candidates also had difficulty deciding if a diagnostic was an actual violation of a CERT rule, even when they fully understood the code. Two analyst candidates incorrectly marked diagnostic pair 26 as false because the diagnostic text referred to a MISRA rule that had been violated, and the analyst candidates had not considered that MISRA was not authoritative for the purpose of this test [MISRA 2004].

Some diagnostics were incorrectly marked true because they indicated portability problems rather than security problems. For instance, diagnostic pair 24 indicated code that was not portable across different platforms but was perfectly secure when run on its intended platform. The code depended on specific integer sizes, which are guaranteed by particular implementations of C, but not by the $\mathrm{C}$ standard.

There were also many errors caused by insufficient whole-program analysis. Interestingly, all of the cases where the analyst candidate disagreed with the group arose because the administrator failed to perform sufficient whole-program analysis. One (or more) of the analyst candidates performed a more comprehensive analysis on a diagnostic, causing them to come to a different conclusion and convince the group that the administrator's answer key was incorrect.

These scores lead us to conclude that analyst candidates without special training are not qualified to produce accurate or consistent analysis results. This may be because of the analyst candidates' lack of knowledge or experience or because of poor testing conditions. Furthermore the test should be specified more rigorously so that analyst candidates are not unduly influenced by external authorities, such as MISRA.

Rules that require whole-program analysis are also problematic because whole-program analysis is prohibitively expensive, and analysis costs scale exponentially with program size. Many rules try to not require whole-program analysis, but some cannot be enforced without it. For instance, checking for memory leaks requires detailed knowledge of the entire codebase. Evaluating these rules only in the context of a particular function can result in false positives being identified as actual violations. In many cases, the developer may need to provide the evidence that these are not true violations. 


\section{Conformance Testing}

\subsection{Introduction}

In general, objective third-party evaluation of a product provides confidence and assurance that the product conforms to a specific standard. The CERT SCALe assesses a software system, determines if it conforms to a CERT secure coding standard, and provides evidence to that effect. The services are performed under a service agreement.

Conformance testing by a recognized and respected organization such as the CERT Program ensures the impartiality of the assessment, ensures fair and valid testing processes, and fosters confidence and acceptance of the software by consumers in the public and private sectors.

According to the results of a recent survey conducted for the Independent Association of Accredited Registrars (IAAR), the main motives organizations cited for obtaining a third-party certification of conformance to a quality standard were "customer mandate” (29\%), "competitive pressure or advantage” (17\%), “continuous improvement based on customer requirements” (16\%), and "improve quality" (14\%). Less frequently cited were "implementation and control of best practice” (10\%) and "corporate mandate” (9\%). "Reduce cost," "risk management," and "legal reasons" were each cited by $1 \%$ of respondents [ANAB 2008].

For many organizations, product certification yields financial benefits because of cost reduction and new sources of revenue. Among respondents to the IAAR survey, $86 \%$ of companies certified in quality management realized a positive return on investment (ROI). An ROI of more than $10 \%$ was reported by $26 \%$ of respondents to the survey.

While undergoing third-party audits to become certified may be voluntary, for many organizations there are compelling reasons to do so:

- improve the efficiency and effectiveness of operations

- $\quad$ satisfy customer requirement

- $\quad$ satisfy contractual, regulatory, or market requirement

- instill organizational discipline

- $\quad$ demonstrate to shareholders, regulators, and the public that a software product has been audited

- instill customer confidence

- $\quad$ identify issues that may be overlooked by those inside the organization, providing fresh internal improvement strategies

Common elements of conformance assessment include impartiality, confidentiality, complaints and appeals, and information disclosure policy. 


\subsubsection{Impartiality}

The CERT Program resides within Carnegie Mellon University's Software Engineering Institute, a federally funded research and development center. The SEI and the CERT Program are frequently called upon to provide impartial third-party assessments.

\subsubsection{Complaints and Appeals}

CERT records and investigates complaints received from customers or other parties and, when warranted, takes corrective action. The CERT Program monitors the results to ensure the effectiveness of corrective actions.

It is not uncommon for a software developer to dispute a finding as being a false positive. In these cases, the software developer is required to provide evidence to the CERT Program that the finding is a false positive. The CERT Program then reviews this evidence and either corrects the finding or refutes the evidence. In cases where the coding construction is determined to be a violation of a secure coding rule but can be demonstrated to present no vulnerability because of architectural, design, or deployment constraints, the developer may request, and will be granted, a deviation.

\subsubsection{Information Disclosure Policy}

The CERT Program holds proprietary information (such as source code) in the strictest confidence and maintains its confidentiality by using at least as much care as the client uses to maintain the confidentiality of its own valuable proprietary and confidential information. The CERT Program will not disclose this information to employees other than to those whose official duties require the analysis of the source code. The CERT Program will not disclose proprietary information to any third party without the prior written consent of the customer. All obligations of confidentiality survive the completion of the conformance assessment process.

The CERT Program may publish company-specific information in aggregate form and without attribution to source.

\subsection{CERT SCALe Seal}

Developers of software that has been determined by the CERT Program as conforming to a secure coding standard may use the seal shown in Figure 6 to describe the conforming software on the developer's website. The seal must be specifically tied to the software passing conformance testing and not applied to untested products, the company, or the organization.

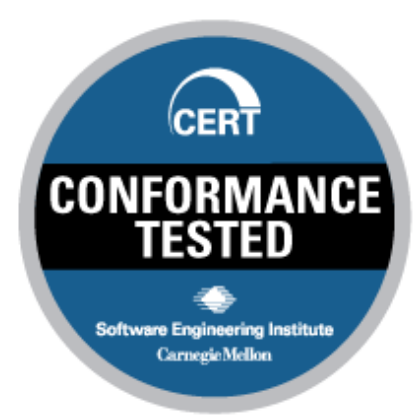

Figure 6: CERT SCALe Seal 
Except for patches that meet the criteria below, any modification of software after it is designated as conforming voids the conformance designation. Until such software is retested and determined to be conforming, the new software cannot be associated with the CERT SCALe seal.

Patches that meet all three of the following criteria do not void the conformance designation:

- The patch is necessary to fix a vulnerability in the code or is necessary for the maintenance of the software.

- The patch does not introduce new features or functionality.

- The patch does not introduce a violation of any of the rules in the secure coding standard to which the software has been determined to conform.

Use of the CERT SCALe seal is contingent upon the organization entering into a service agreement with Carnegie Mellon University and upon the software being designated by the CERT Program as conforming.

\subsection{CERT SCALe Service Agreement}

Organizations seeking SCALe conformance testing will abide by the SCALe policies and procedures required by the SCALe Service Agreement. Organizations submitting software code for conformance testing will follow these basic processes:

1. A service agreement must be fully executed by the organization and Carnegie Mellon University's Software Engineering Institute before conformance testing begins.

2. The CERT Program evaluates the source code of the software against the identified CERT secure coding standard(s), specified in the statement of work, using the identified tools and procedures and provides an initial conformance test report to the client that catalogues all rule violations found as a result of the SCALe evaluation.

3. From receipt of the initial conformance test report, the client has 180 days to repair nonconforming code and/or prepare documentation that supports the conclusion that identified violations do not present known vulnerabilities and resubmit the software and any deviation requests for a final evaluation of the software against the specified CERT secure coding standard(s).

4. The CERT Program will evaluate any deviation requests and reevaluate the software against the specified CERT secure coding standard(s) and provide a final conformance test report to the client.

5. Clients are permitted to use the CERT SCALe seal on their website in connection with successful product conformance testing after the product version has passed the applicable conformance test suite(s). Clients may describe the product version as having been determined by The CERT Program to conform to the CERT secure coding standard.

6. Clients whose software passes the conformance testing agree to have their product version listed on the CERT web registry of conforming systems.

\subsubsection{Conformance Certificates}

SCALe validation certificates include the client organization's name, product name, product version, and registration date. Certificates also include a list of applicable guidelines and an 
indication if, for a particular guideline, the source code being tested was determined to be provably conforming or conforming.

\section{Registry of Conforming Products}

The CERT Program will maintain an online certificates registry of systems that conform to CERT secure coding standards at https://www.securecoding.cert.org/registry.

\subsection{SCALe Accreditation}

The CERT Program will not initially seek American National Standards Institute (ANSI), International Organization for Standardization (ISO), or NIST accreditation for SCALe from an accreditation agency. However, The CERT Program will endeavor to implement processes, procedures, and systems that comply with national and international standards. As needed, the program can submit for accreditation by the following agencies:

- $\quad$ ISO/IEC. This agency has published ISO/IEC 65, which provides principles and requirements for the competence, consistency, and impartiality of third-party certification bodies evaluating and certifying products (including services) and processes. The agency has also published ISO/IEC 17025:2005 General Requirements for the Competence of Testing and Calibration Laboratories, which specifies the requirements for sound management and technical competence for the type of tests and calibrations SCALe undertakes. Testing and calibration laboratories that comply with ISO/IEC 17025 also operate in accordance with ISO 9001.

- $\quad$ NIST National Voluntary Laboratory Accreditation Program (NVLAP). NVLAP provides third-party accreditation to testing and calibration laboratories. NVLAP's accreditation programs are established in response to Congressional mandates, administrative actions by the federal government, and requests from private-sector organizations and government agencies.

- $\quad$ NVLAP operates an accreditation system that is compliant with ISO/IEC 17011:2004 Conformity assessment. It provides general requirements for bodies accrediting conformance assessment bodies, which requires that the competence of applicant laboratories be assessed by the accreditation body against all of the requirements of ISO/IEC 17025: 2005 General requirements for the competence of testing and calibration laboratories.

\subsection{Transition}

Transition of SCALe to practice will follow the SEI's transition strategy to grow the concept through engagement with external organizations or SEI partners via a series of deliberate steps. The proof-of-concept phase will occur with a piloting program of SCALe that engages a small number of clients. During this phase, the CERT Program will test and refine processes, procedures, systems, and outputs.

After the pilot phase, the CERT Program will engage a small number of additional organizations that will be licensed to sponsor SCALe laboratories within themselves. Each organization will be licensed to perform the assessment, issue the conformance assessment report, report results to the CERT Program, and be subject to annual quality audits of all processes, procedures, hardware, and software. 


\subsection{Conformance Test Results}

The following sections present example test results of two systems' conformance to CERT secure coding standards, as determined by SCALe analysis.

\subsubsection{System A}

Table 6 shows the flagged nonconformities reported from analysis of the first system. The analysis was performed using four static analysis tools supplemented by manual code inspection. Dynamic analysis was not used.

Table 6: Flagged Nonconformities, System A

\begin{tabular}{|c|c|c|c|c|c|c|}
\hline & Manual & Analyzer A & Analyzer B & Analyzer C & Analyzer D & Total \\
\hline DCL31-C & & & 0 & 705 & & 705 \\
\hline DCL32-C & & & 0 & 119 & 1 & 120 \\
\hline DCL35-C & & & 0 & 51 & & 51 \\
\hline DCL36-C & & & 0 & 19 & & 19 \\
\hline EXP30-C & & & 0 & 3 & & 3 \\
\hline EXP34-C & & & 0 & 54 & 4 & 58 \\
\hline EXP36-C & & & 0 & 24 & 6 & 30 \\
\hline EXP37-C & & & 0 & 49 & & 49 \\
\hline INT31-C & & 1 & 4 & 3,588 & & 3,593 \\
\hline INT35-C & & 6 & 0 & & & 6 \\
\hline FLP34-C & & & 0 & 9 & & 9 \\
\hline FLP36-C & & & 0 & 1 & & 1 \\
\hline ARR30-C & & 4 & 2 & 1 & & 7 \\
\hline STR31-C & & & 3 & & & 3 \\
\hline STR36-C & & & 0 & 6 & & 6 \\
\hline STR37-C & & & 0 & & 1 & 1 \\
\hline STR38-C & & & 7 & & & 7 \\
\hline MEM34-C & & & 0 & & 2 & 2 \\
\hline FIO30-C & & & 0 & 12 & & 12 \\
\hline ENV30-C & & & 0 & & 3 & 3 \\
\hline SIG30-C & 1 & & 0 & & & 1 \\
\hline CON33-C & 1 & & 0 & & & 1 \\
\hline MSC31-C & & & 0 & & 1 & 1 \\
\hline MSC34-C & & & 0 & 587 & & 587 \\
\hline Total & 2 & 11 & 16 & 5,228 & 18 & 5,275 \\
\hline
\end{tabular}

The first column marked "Manual" shows violations that were discovered through manual code inspection, while the four columns marked “Analyzer A,” "Analyzer B," “Analyzer C,” and "Analyzer D" show the number of flagged nonconformities detected by each of the four analysis tools used in this analysis. 
Table 7 shows the results of analysis of the flagged nonconformities by the SCALe analysts and SCALe lead assessor combined.

Table 7: Analysis Results, System A

\begin{tabular}{|l|r|r|r|r|}
\hline & False & True & Unknown & Total \\
\hline DCL31-C & 705 & & & 705 \\
\hline DCL32-C & 2 & 2 & 116 & 120 \\
\hline DCL35-C & & 2 & 49 & 51 \\
\hline DCL36-C & 19 & & & 19 \\
\hline EXP30-C & 2 & 1 & & 3 \\
\hline EXP34-C & 2 & 4 & 52 & 58 \\
\hline EXP36-C & 4 & 4 & 22 & 30 \\
\hline EXP37-C & 4 & 1 & 44 & 49 \\
\hline INT31-C & 1,999 & & 1,594 & 3,593 \\
\hline INT35-C & & 6 & & 6 \\
\hline FLP34-C & 7 & 2 & & 9 \\
\hline FLP36-C & 1 & & & 1 \\
\hline ARR30-C & 7 & & & 7 \\
\hline STR31-C & 3 & & & 3 \\
\hline STR36-C & 4 & 2 & & 6 \\
\hline STR37-C & & 1 & & 1 \\
\hline STR38-C & 3 & 4 & & 7 \\
\hline MEM34-C & 2 & & & 2 \\
\hline FIO30-C & 12 & & & 12 \\
\hline ENV30-C & 3 & & & \\
\hline SIG30-C & & 1 & & \\
\hline CON33-C & & 1 & & \\
\hline MSC31-C & & 1 & & \\
\hline
\end{tabular}

The "False" and "True" columns document the number of flagged nonconformities that were determined to be false and true positives, respectively. Normally it is sufficient to stop after finding one true positive, but in cases with a small number of flagged nonconformities, all the results were evaluated to collect data about the true positive and flagged nonconformity rates for the analyzer checkers. Flagged nonconformities that were not evaluated are marked as "Unknown."

This particular software system violated at least 15 of the CERT C secure coding rules. In nine other cases, manual analysis eliminated possible rule violations as false positives. 


\subsubsection{System B}

The second system was also evaluated by four static analysis tools supplemented by manual inspection. Two of the tools (analyzers A and B) were also used in the analysis of system A. The other two analyzers were used for the first time in the analysis of system B. Table 8 shows the flagged nonconformities found from the analysis of the second system.

Table 8: $\quad$ Flagged Nonconformities, System B

\begin{tabular}{|c|c|c|c|c|c|c|}
\hline & Manual & Analyzer B & Analyzer C & Analyzer E & Analyzer F & Total \\
\hline ARR30-C & & & 17 & 1 & & 18 \\
\hline ARR36-C & 2 & & 106 & & & 108 \\
\hline DCL35-C & & & 47 & & & 47 \\
\hline DCL36-C & & & 2 & & & 2 \\
\hline EXP30-C & & & 2 & & & 2 \\
\hline EXP33-C & & & 308 & 1 & 25 & 334 \\
\hline EXP34-C & & 21 & 483 & 68 & 49 & 621 \\
\hline EXP36-C & & & 109 & & & 109 \\
\hline EXP37-C & & & 40 & & & 40 \\
\hline EXP40-C & & & 20 & & & 20 \\
\hline FIO30-C & & & 2 & 6 & & 8 \\
\hline FLP34-C & & & 324 & & & 324 \\
\hline FLP35-C & & & 9 & & & 9 \\
\hline INT31-C & & 8 & 7,562 & 5 & 2 & 7,577 \\
\hline INT32-C & & & & 7 & 2 & 9 \\
\hline МЕM30-С & & & & 1 & 2 & 3 \\
\hline MEM31-C & & 10 & 7 & & 4 & 21 \\
\hline MEM33-C & & & 4 & & & 4 \\
\hline MEM34-C & & & 1 & & & 1 \\
\hline MSC34-C & & & 362 & & & 362 \\
\hline PRE30-C & & & 4 & & & 4 \\
\hline PRE31-C & & & 11 & & & 11 \\
\hline STR30-C & & & 11 & & & 11 \\
\hline STR31-C & & 1 & & 5 & 44 & 50 \\
\hline STR32-C & & 1 & & 10 & & 11 \\
\hline STR33-C & & 1 & & & & 1 \\
\hline Total & 2 & 42 & 9,431 & 104 & 128 & 9,707 \\
\hline
\end{tabular}


Table 9 shows the results of analysis of the flagged nonconformities by the SCALe analysts and SCALe lead assessor combined. Unfortunately, this analysis was not completed. Where all flagged nonconformities for a rule were unknown, the nonconformities have not been evaluated.

Table 9: Analysis Results, System B

\begin{tabular}{|c|c|c|c|c|c|}
\hline & False & Suspicious & True & Unknown & Total \\
\hline ARR30-C & & & & 18 & 18 \\
\hline ARR36-C & & & 2 & 106 & 108 \\
\hline DCL35-C & & & & 47 & 47 \\
\hline DCL36-C & & & 2 & & 2 \\
\hline EXP30-C & 2 & & & & 2 \\
\hline EXP33-C & 5 & & & 329 & 334 \\
\hline EXP34-C & 13 & & 3 & 605 & 621 \\
\hline EXP37-C & & & & 40 & 40 \\
\hline EXP40-C & & & & 20 & 20 \\
\hline FIO30-C & 3 & 2 & 3 & & 8 \\
\hline FLP35-C & & & & 9 & 9 \\
\hline INT31-C & 603 & & 2 & 6,971 & 7,576 \\
\hline INT32-C & & & & 9 & 9 \\
\hline MEM30-C & 3 & & & & 3 \\
\hline MEM31-C & & & & 21 & 21 \\
\hline MEM33-C & & & 4 & & 4 \\
\hline MEM34-C & & & 1 & & 1 \\
\hline MSC34-C & 326 & & 36 & & 362 \\
\hline PRE30-C & 4 & & & & 4 \\
\hline PRE31-C & & & & 11 & 11 \\
\hline STR30-C & & & & 11 & 11 \\
\hline STR31-C & & & 1 & 50 & 51 \\
\hline STR32-C & & & & 11 & 11 \\
\hline STR33-C & & & 1 & & 1 \\
\hline
\end{tabular}

Based on our experience with analyzing system A, we added a new category of "suspicious.” This category includes flagged nonconformities that could not easily be proven to be either true or false positives. This was frequently the case for dereferencing null pointers, for example, where the pointer dereferences were unguarded but it was difficult to prove that the pointer was never null without performing whole-program analysis. Suspicious violations are treated as false positives in that they will not result in a system failing conformance testing and will not stop the analyst from analyzing other flagged nonconformities reported against the same coding rule. These are reported as suspicious so that the developer can examine these flagged nonconformities and take appropriate measures.

Overall, system B had considerably more flagged nonconformities than system A, a significant number of which have already been determined to be true positives. 


\section{Related Efforts}

This section describes related conformance assessment activities in today’s marketplace.

\subsection{Veracode}

Veracode's ${ }^{14}$ Risk Adjusted Verification Methodology allows organizations developing or procuring software to measure, compare, and reduce risks related to application security. Veracode uses static binary analysis, dynamic analysis, and manual penetration testing to identify security flaws in software applications. The basis for the VERAFIED security mark is the Security Quality Score (SQS). SQS aggregates the severities of all security flaws found during the assessment and normalizes the results to a scale of 0 to 100 . The score generated by each type of assessment is then mapped to the application's business criticality (assurance level), and those applications that reach the highest rating earn the VERAFIED security mark.

\subsection{ICSA Labs}

ICSA Labs, ${ }^{15}$ an independent division of Verizon Business, has been providing independent, third-party product assurance for end users and enterprises for 20 years. ICSA Labs says they provide "vendor-neutral testing and certification for hundreds of security products and solutions for many of the world's top security product developers and service providers” [Cybertrust 2010]. ICSA Labs provides services in three areas:

- Consortium Operations, Security Product Testing, and Certification Programs

- $\quad$ Custom Testing Services

- $\quad$ Accredited Government Testing Services

ICSA Labs is ISO 17025:2005 accredited and ISO 9001:2008 registered.

\subsection{SAIC Accreditation and Certification Services}

SAIC (Science Applications International Corporation ) ${ }^{16}$ provides security content automation protocol (SCAP) testing and monitoring of systems for security issues such as software deficiencies, configuration issues, and other vulnerabilities. The testing helps ensure that a computer's configuration is within guidelines set by the Federal Desktop Core Configuration. Notably, they became an accreditation body under the NIST accreditation to perform SCAP.

\subsection{The Open Group Product Certification Services}

The Open Group ${ }^{17}$ has developed and operates an industry-based product certification program in several areas, including UNIX, CORBA, POSIX, and LDAP. They have developed and currently

\footnotetext{
$14 \mathrm{http}: / / \mathrm{www} \cdot \mathrm{veracode} . \mathrm{com} /$

15 http://www.icsalabs.com/

$16 \mathrm{http} / / / \mathrm{ww}$.saic.com/infosec/testing-accreditation/scap.html

17 http://www.opengroup.org/consortia_services/certification.htm
} 
maintain conformance test suites for multiple technologies, including those listed above, the $\mathrm{X}$ Window System, Motif, Digital Video Broadcasting Multimedia Home Platform, Secure Electronic Transactions (SET), Common Data Security Architecture (CDSA), and Linux [Open Group 2010].

The Open Group product certification program provides formal recognition of a product's conformance to an industry standard specification. This allows suppliers to make and substantiate clear claims of conformance to a standard and allows buyers to specify and successfully procure conforming products that interoperate [Open Group 2010].

The Open Group's product certification programs are based on a supplier's claim of conformance; testing provides an indicator of conformance. Suppliers typically use test suites to establish confidence that their product conforms. To achieve certification, the supplier must provide a warranty of conformance ensuring the following [Open Group 2010]:

- $\quad$ products conform to an industry standard specification

- $\quad$ products remain conformant throughout their lifetimes

- the product will be fixed in a timely manner if there is a nonconformance

The Open Group acts as the independent certification authority for industry-based certification programs. As the certification authority, their web-based conformance testing system is tailored to guide suppliers through the process of certifying a product [Open Group 2010]. 


\section{Future Work and Summary}

\subsection{Future Work}

Work is continuing on the development of secure coding standards for $\mathrm{C}++$, Java, and other programming languages. As these standards are completed and adequate tooling becomes available, SCALe will be extended to support conformance testing against these secure coding standards.

The CERT Program will also expand SCALe's operational capability, including integrating additional commercial and research analyzers into the SCALe laboratory environment. This process includes acquiring tools, creating a mapping between diagnostics generated by the tool and CERT secure coding standards, and automating the processing of these diagnostics.

In addition to the use of acceptance sampling plans based on the lot tolerance percent defective, other techniques can be researched for use when greater amounts of data from conformance testing are available. These techniques, including Bayesian methods, may enable even more informed decisions for the stopping rules related to the investigation of flagged nonconformities for false positives. It is anticipated that such analysis will eventually be granular down to the flagged nonconformity and help further reduce the sample size of flagged nonconformities to be investigated.

Additionally, a number of techniques can be explored to characterize the performance of each of the security checker tools in terms of each tool's

- $\quad$ proportion of false positives to true positives

- $\quad$ ability to find certain classes of true positives that are not discovered by other analyzers

Given this information, more informed decisions can be made within each security analysis event in terms of which checker tools to employ. The SCALe lead assessor would discontinue the use of specific checkers that have a high proportion of false positives and little, if any, contribution to the identification of true positives above and beyond what the other checker tools are capable of finding.

\subsection{Summary}

Growing numbers of vulnerability reports and reports of software exploitations demand that underlying issues of poor software quality and security be addressed. Conformance with CERT secure coding standards is a measure of software security and quality that provides an indication of product security. SCALe provides a defined, repeatable process for conformance testing of software systems. Conformance testing against the CERT secure coding standard should help establish a market for secure software by allowing vendors to market software quality and security and also enable consumers to identify and purchase conforming products. 


\section{Bibliography}

URLs are valid as of the publication date of this document.

\section{[ANAB 2008]}

ANSI-ASQ National Accreditation Board (ANAB). The Third-Party Process: Waste of Resources or Added Value? ANAB, 2008.

http://www.anab.org/media/9593/valueofaccreditedcertification.pdf

\section{[Ashling Microsystems]}

Ashling Microsystems. Ashling Microsystems. http://www.ashling.com/technicalarticles/APB201TestbedDescription.pdf, 2010.

\section{[CERT 2010a]}

CERT. The CERT C Secure Coding Standard, Version 2.0. Software Engineering Institute, Carnegie Mellon University, 2010. https://www.securecoding.cert.org/confluence/X/HQE

\section{[CERT 2010b]}

CERT. The CERT C++ Secure Coding Standard. Software Engineering Institute, Carnegie Mellon University, 2010. https://www.securecoding.cert.org/confluence/x/fQI

\section{[CERT 2010c]}

CERT. The CERT Oracle Secure Coding Standard for Java. Software Engineering Institute, Carnegie Mellon University, 2010. https://www.securecoding.cert.org/confluence/x/Ux

\section{[Christey 2007]}

Christey, Steve \& Martin, Robert A. Vulnerability Type Distributions in CVE, Version: 1.1. MITRE, 2007. http://cve.mitre.org/docs/vuln-trends/vuln-trends.pdf

\section{[Cybertrust 2010]}

Cybertrust. About ICSA Labs. http://www.icsalabs.com/about-icsa-labs, 2010.

\section{[Dannenberg 2010]}

Dannenberg, Roger B.; Dormann, Will; Keaton, David; Seacord, Robert C.; Svoboda, David; Volkovitsky, Alex; Wilson, Timothy; \& Plum, Thomas. “As-If Infinitely Ranged Integer Model,” 91-100. Proceedings 2010 IEEE 21st International Symposium on Software Reliability

Engineering (ISSRE 2010), San Jose, CA, Nov. 2010. IEEE 2010.

\section{[Heffley 2004]}

Heffley, J. \& Meunier, P. “Can Source Code Auditing Software Identify Common Vulnerabilities and Be Used to Evaluate Software Security?” Proceedings of the 37th Annual Hawaii International Conference on System Sciences (HICSS'04) - Track 9 - Volume 9. Island of Hawaii, January 2004. IEEE Computer Society, 2004. 


\section{[IEC 2006]}

International Electrotechnical Commission (IEC). Analysis Techniques for System Reliability Procedure for Failure Mode and Effects Analysis (FMEA), 2nd ed. (IEC 60812). IEC, January 2006.

\section{[IEEE Std 610.12 1990]}

IEEE. IEEE Standard Glossary of Software Engineering Terminology (Std. 610.12-1990). IEEE, 1990.

\section{[ISO/IEC 2004]}

International Organization for Standardization, International Electrotechnical Commission (ISO/IEC). ISO/IEC 17000:2004 Conformity Assessment — Vocabulary and General Principles, 1st edition. ISO, 2004.

\section{[ISO/IEC 2005]}

International Organization for Standardization, International Electrotechnical Commission (ISO/IEC). ISO/IEC 9899:1999 Programming Languages—C. ISO, 2005.

http://www.open-std.org/JTC1/SC22/wg14/www/docs/n1124.pdf

\section{[ISO/IEC 2007]}

International Organization for Standardization, International Electrotechnical Commission (ISO/IEC). ISO/IEC TR 24731-1:2007 Extensions to the C Library - Part I: Bounds-checking interfaces. ISO, August 2007.

\section{[ISO/IEC 2010a]}

International Organization for Standardization, International Electrotechnical Commission (ISO/IEC). ISO/IEC TR 24731-2:2010 Extensions to the C Library — Part II: Dynamic

Allocation Functions. ISO, 2010.

\section{[ISO/IEC 2010b]}

International Organization for Standardization, International Electrotechnical Commission (ISO/IEC). Programming Languages-C++, Final Committee Draft, ISO/IEC JTC1 SC22 WG21 N3092. ISO, March 2010.

\section{[ISO/IEC 2011]}

International Organization for Standardization. ISO/IEC 9899:2011. Information Technology — Programming Languages - C. International Organization for Standardization, 2011.

\section{[Landis 1977]}

Landis, J. R. \& Koch, G. G. “The Measurement of Observer Agreement for Categorical Data.” Biometrics 33 (1977): 159-174.

\section{[Landwehr 2008]}

Landwehr, C. IARPA STONESOUP Proposers Day. IARPA, 2008.

http://www.iarpa.gov/Stonesoup_Proposer_Day_Brief.pdf 


\section{[Long 2012]}

Long, Fred; Mohindra, Dhruv; Seacord, Robert C.; Sutherland, Dean F.; \& Svoboda, David. The CERT Oracle Secure Coding Standard for Java. Addison-Wesley Professional, 2012.

\section{[MISRA 2004]}

Motor Industry Software Reliability Association (MISRA). MISRA-C:2004: Guidelines for the Use of the C Language in Critical Systems. MIRA, 2004.

\section{[Okun 2009]}

Okun, V.; Gaucher, R.; \& Black, P. E., eds. Static Analysis Tool Exposition (SATE) 2008 (NIST

Special Publication 500-279). NIST, 2009.

\section{[Open Group 2010]}

The Open Group. Collaboration Services.

http://www.opengroup.org/consortia_services/certification.htm, 2010.

\section{[Plum 2005]}

Plum, Thomas \& Keaton, David M. "Eliminating Buffer Overflows, Using the Compiler or a Standalone Tool," 75-81. Proceedings of the Workshop on Software Security Assurance Tools, Techniques, and Metrics. Long Beach, CA, November 2005. U.S. National Institute of Standards and Technology (NIST), 2005.

\section{[Plum 2009]}

Plum, Thomas \& Seacord, Robert C. ISO/IEC JTC 1/SC 22/WG14/N1350 - Analyzability. International Standards Organization (ISO), 2009.

http://www.open-std.org/jtc1/sc22/wg14/www/docs/n1350.htm

\section{[Saltzer 1975]}

Saltzer, Jerome H. \& Schroeder, Michael D. "The Protection of Information in Computer Systems.” Proceedings of the IEEE 63, 9 (September 1975): 1278-1308.

\section{[Seacord 2003]}

Seacord, R. C.; Plakosh, D.; \& Lewis, G. A. Modernizing Legacy Systems: Software Technologies, Engineering Process and Business Practices. Addison-Wesley Longman Publishing Co., Inc., 2003.

\section{[Seacord 2005]}

Seacord, R. C. Secure Coding in C and C++ (SEI Series in Software Engineering). AddisonWesley Professional, 2005.

\section{[Seacord 2008]}

Seacord, R. The CERT C Secure Coding Standard. Addison-Wesley Professional, 2008.

\section{[Stephens 2001]}

Stephens, Kenneth S. The Handbook of Applied Acceptance Sampling. ASQ Quality Press, 2001. 
[Takanen 2008]

Takanen, A.; DeMott, J.; \& Miller, C. Fuzzing for Software Security Testing and Quality Assurance. 1. Artech House, Inc., 2008.

[von Eye 2006]

von Eye, Alexander \& Mun, Eun Young. Analyzing Rater Agreement: Manifest Variable Methods. Lawrence Erlbaum Associates, 2006. 


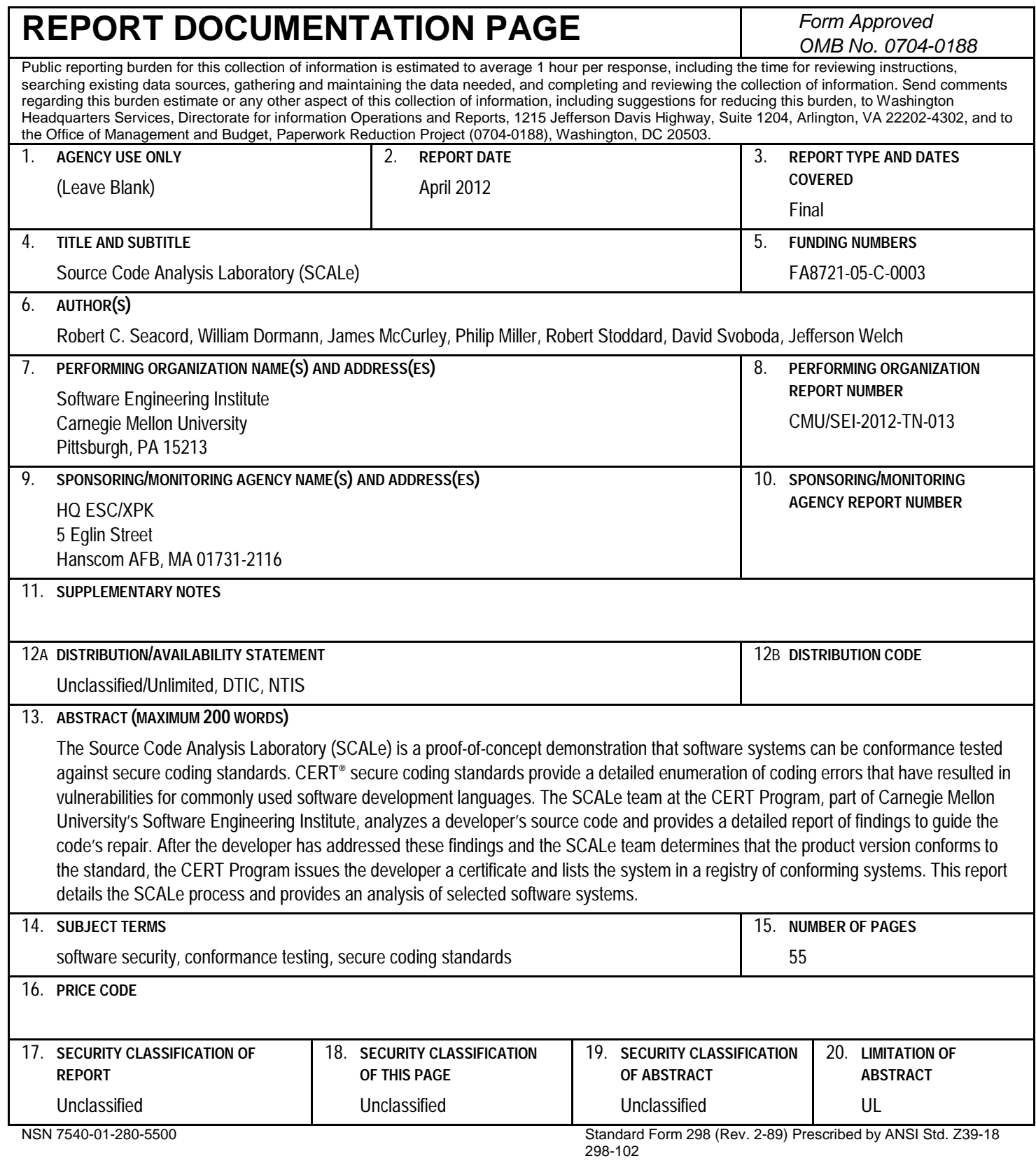

